\title{
Grid-dependence study for simulating propeller crashback using large-eddy simulation with immersed boundary method
}

\author{
Fei Liao, Xiaolei Yang *, Shizhao Wang, Guowei He \\ State Key Laboratory of Nonlinear Mechanics, Institute of Mechanics, Chinese Academy of Sciences, Beijing, 100190, China \\ School of Engineering Sciences, University of Chinese Academy of Sciences, Beijing 100049, China
}

\section{A R T I C L E I N F O}

\section{Keywords:}

Grid-dependence

Propeller

Crashback

Large-eddy simulation

Immersed boundary method

\begin{abstract}
A B S T R A C T
Simulating the flow around a propeller using large-eddy simulation (LES) with the immersed boundary (IB) method is challenging and computationally expensive. In this work, we carry out the grid-dependence study of LES with IB for simulating a propeller in crashback mode using four sets of gradually refined grids. The simulation results show that the grid-resolution requirements for accurately predicting different flow quantities are different. Specifically, it is found that the side-force coefficient and the averaged streamwise velocity are less sensitive to grid resolutions as compared with the thrust force coefficient and the turbulence kinetic energy, respectively. Furthermore, it is found that the computed results in the near wake region and the region around the blade are more sensitive to grid resolutions as compared with the far wake region, where the predictions from the four different grids are similar to each other. This suggests that a coarse grid simulation is adequate if only the far wake region is of interest, while a fine grid simulation is required if one cares about the flow around the blade and the flow in the near wake region.
\end{abstract}

\section{Introduction}

Simulating the turbulent flow around a real-world propeller using computational fluid dynamics (CFD) is challenging due to high Reynolds number, strong unsteadiness, and complex geometric and moving boundaries. Turbulence models of different fidelity and computational cost, such as large-eddy simulation (LES) and Reynoldsaveraged Navier-Stokes (RANS) simulation, are often employed in simulating such complex flows. LES, which is more accurate than RANS in predicting the unsteadiness of turbulent flows has been widely used in the simulation of propeller flows (Vysohlid and Mahesh, 2006; Kang et al., 2012, 2014; Sotiropoulos and Yang, 2014; Balaras et al., 2015; Kumar and Mahesh, 2017; Keller et al., 2018).

Simulating the complex geometric boundary of rotating blades poses a great challenge on computational methods based on body-fitted grids. The immersed boundary method, which is based on non-body-fitted grids and does not require complicated body-fitted grids, and grid remeshing for moving boundary problems, has been successfully applied to various engineering problems (Sotiropoulos and Yang, 2014; Huang and Tian, 2019; Griffith and Patankar, 2020; Roy et al., 2020). To simulate flows with three-dimensional complex geometries, the immersed boundary method requires similar grid spacings in all three directions, and thus its grid requirement is often higher than the computational methods based on body-fitted grids, which can become very challenging for flows at high Reynolds number. Using wall models can alleviate the grid requirement in the near wall region, and significantly enhance the capability of the immersed boundary method for simulating complex turbulent flows at high Reynolds number (Tessicini et al., 2002; Roman et al., 2009; Kang, 2015; Shi et al., 2019; Ma et al., 2019). To the best of our knowledge, only a few studies applied the immersed boundary method to simulate turbulent flows over a propeller (Kang et al., 2012; Balaras et al., 2015; Posa and Balaras, 2018; Posa et al., 2019a,b; Brehm et al., 2016). The accuracy, robustness and efficiency of the immersed boundary method with wall model for simulating flows over a propeller still needs to be examined. Particularly it is not clear about the grid sensitivity of different quantities predicted by a wall-modeled LES.

LES of a propeller is very computationally expensive. Balaras et al. (2015) simulated the INSEAN E1619 propeller under openwater condition using 3.2 billion grid nodes. Posa and Balaras (2018) simulated a submarine in self-propelled configuration using 3.5 billion grid nodes with 2048 compute cores for 6 million CPU hours. Kumar and Mahesh (2017) investigated wake instabilities of the DTMB 4381 propeller using 181 million grid cells and 10668 computational time steps per rotation on 2048 processors. Posa et al. (2019a) adopted 840 million grid nodes for LES of a submarine propeller using 1024 cores for about

\footnotetext{
* Corresponding author at: State Key Laboratory of Nonlinear Mechanics, Institute of Mechanics, Chinese Academy of Sciences, Beijing, 100190, China. E-mail address: xyang@imech.ac.cn (X. Yang).
} 
1.5 million CPU hours, which are about 2 months clock time. In Posa et al. (2019b), Posa et al. investigated the wake feature of the INSEAN E1619 propeller in the downstream of a rudder using 1.7 billion grid nodes, 1024 compute cores and 6 million CPU hours per case, which takes about 8 months clock time for a case.

Grid-independent solutions are expected in LES of turbulent flows. Large-scale, energy-containing eddies are directly resolved by LES, while the contributions from subgrid-scale eddies are modeled using different subgrid-scale (SGS) models (Smargorinsky, 1963; Germano et al., 1991; Nicoud and Ducros, 1999). For canonical flow problems at low Reynolds number (Wang and Zhang, 2011), grid-convergent results can be obtained without too much difficulty. On the other hand, for real-life turbulent flow problems at high Reynolds number (Wang et al., 2016; Yang and Sotiropoulos, 2019), sometimes it is not even feasible to carry out grid-convergence studies. Eddies of smaller scale develop as one refines the grid, such that the statistics of the resolved flow statistics are not necessarily the same for two grids of different resolutions (Toosi, 2019). Furthermore, the grid independence of LES results may depend on the quantities of interest, which might be easier to achieve for those which are strong functions of large-scale eddies with little dependence on subgrid scales.

Propeller can operate in forward and crashback conditions. The forward mode is the normal operational condition. The crashback of propeller (Vysohlid and Mahesh, 2006, 2007; Chang et al., 2008a; Verma et al., 2012; Jang and Mahesh, 2013; Kumar and Mahesh, 2016) is an off-design condition, in which the propeller is rotating in reverse direction to slow down the advancing vehicle. During the crashback mode of operation, the blades experience significantly higher loads in both axial and transverse directions with the flow featured by a large amount of detached eddies. Understanding its fluid dynamics is important in terms of both practical applications and fundamental studies of complex turbulent flows. It is difficult for the RANS (ReynoldsAveraged Navier-Stokes) method to simulate propeller in crashback mode because of massive flow separations. LES has been employed in the literature for simulating propeller in crashback. For instance, Verma et al. (2012) investigated the effect of an upstream hull on the side force of a propeller in crashback mode using 7.3 million grid cells. Jang and Mahesh (2013) employed two grids of grid number 7.7 million and 19.3 million to probe into the mechanism for the high-amplitude and the low-amplitude events in thrust and side-forces for different advance ratios. Although simulation of a propeller in crashback mode is less computationally expensive than that in forward mode, as pointed out by Posa et al. (2019a), simulation of a propeller in crashback mode is still challenging especially for wall-modeled LES.

To address the above issue, in this work we carry out wall-modeled LES of a propeller in crashback mode for four different grids with number of grid nodes 14 million, 47 million, 87 million and 179 million. The curvilinear immersed boundary method (CURVIB) with the wall model (Ge and Sotiropoulos, 2007), which has been successfully applied to industrial and environmental turbulent flows (Kang et al., 2014; Khosronejad et al., 2016; Yang et al., 2017), is employed for modeling the rotating blades and other structures. The dynamic subgrid-scale model is employed for modeling unresolved subgrid scales. The thin boundary layer equation without the pressure gradient term is employed as the wall model. We compare the forces, instantaneous flow field, time-averaged flow field and phase-averaged flow field from simulations using four different grids. The present research will be helpful for people to determine the most economic grid resolutions based on the quantities of interest.

This rest of the paper is organized as follows. The flow solver is briefly described in Section 2. Then, the simulation setups are given in Section 3. Next, the simulation results are presented and analyzed in Section 4. At last, conclusions from this work are drawn in Section 5.

\section{Flow solver}

The virtual flow simulator (VFS-Wind) Yang et al. (2015) is employed in this work for LES of the flow around a propeller. The governing equations are the three-dimensional unsteady spatially filtered incompressible Navier-Stokes equations in non-orthogonal, generalized curvilinear coordinates shown as follows:

$$
\begin{aligned}
& J \frac{\partial U^{j}}{\partial \xi^{j}}=0 \\
& \frac{1}{J} \frac{\partial U^{i}}{\partial t}=\frac{\xi_{l}^{i}}{J}\left(-\frac{\partial}{\partial \xi^{j}}\left(U^{j} u_{l}\right)-\frac{1}{\rho} \frac{\partial}{\partial \xi^{j}}\left(\frac{\xi_{l}^{j} p}{J}\right)+\frac{\mu}{\rho} \frac{\partial}{\partial \xi^{j}}\left(\frac{g^{j k}}{J} \frac{\partial u_{l}}{\partial \xi^{k}}\right)-\frac{1}{\rho} \frac{\partial \tau_{l j}}{\partial \xi^{j}}+f_{l}\right)
\end{aligned}
$$

where $x_{i}$ and $\xi^{i}$ are the Cartesian and curvilinear coordinates, respectively, $\xi_{l}^{i}=\partial \xi^{i} / \partial x_{l}$ are the transformation metrics, $J$ is the Jacobian of the geometric transformation, $u_{i}$ is the $i$ th component of the velocity vector in Cartesian coordinates, $U^{i}=\left(\xi_{m}^{i} / J\right) u_{m}$ is the contravariant volume flux, $g^{j k}=\xi_{l}^{j} \xi_{l}^{k}$ are the components of the contravariant metric tensor, $\rho$ is the density, $\mu$ is the dynamic viscosity, $p$ is the pressure, $f_{l}(l=1,2,3)$ are the forcing terms representing the effects of the immersed bodies on the fluid. $\tau_{i j}$ represents the anisotropic part of the subgrid-scale stress tensor, which is modeled by the dynamic eddy viscosity subgrid-scale stress model,

$\tau_{i j}-\frac{1}{3} \tau_{k k} \delta_{i j}=-2 v_{t} \tilde{S}_{i j}$

where $\tilde{S}_{i j}$ is the filtered strain-rate tensor. The modeled eddy viscosity is calculated by

$v_{t}=C \Delta^{2}|\tilde{S}|$

where $C$ is calculated dynamically using the procedure of Germano et al. (1991), $|\tilde{S}|=\sqrt{2 \tilde{S}_{i j} \tilde{S}_{i j}}$ and $\Delta=J^{-1 / 3}$ is the filter size, where $J^{-1}$ is the cell volume.

The CURVIB method is employed to take into account the effect of the solid wall on the surrounding flow, in which the governing equations are discretized and solved on non-body-fitted grids. The geometry of the solid body is discretized using unstructured triangular meshes and immersed into the background grid, which indicates that the background grids do not coincide with the immersed triangular meshes and the boundary conditions are not applied directly but through forcing terms on the background grids. Specifically in the CURVIB method, the velocities at the IB nodes, which are located in the immediate vicinity of the immersed boundary in the fluid, are reconstructed from neighboring fluid nodes. For DNS and wall-resolved LES, the velocities at the IB nodes are interpolated linearly in the wall-normal direction. For wall-modeled LES, the velocities at the IB nodes are reconstructed through a wall model. In the employed wall model, we solve the simplified thin boundary layer equation (TBLE) to obtain the tangential velocity at the IB nodes, which is in the following form:

$\frac{\partial}{\partial y}\left(\left(v+v_{t}\right) \frac{\partial u}{\partial y}\right)=0$

where $y$ is the coordinate in the wall-normal direction, $u$ is the wallparallel velocity, and $v_{t}$ is the eddy viscosity calculated by the mixing length model shown as follows:

$v_{t}=\kappa y u_{*} D_{f}$,

where $u_{*}$ is the friction velocity, the Karman constant $\kappa$ equals 0.4 , and $D_{f}=\left[1-\exp \left(-y u_{*} / \nu A\right)\right]^{2}$ with $A=19$ is the van Driest damping function, and obtain the wall normal velocity by linear interpolations. More details about the CURVIB method can be found in Ge and Sotiropoulos (2007), Borazjani et al. (2008) and Khosronejad et al. (2011).

The governing equations are discretized in space using a secondorder accurate central differencing scheme, and integrated in time using the fractional step method. An algebraic multigrid acceleration 
Table 1

Mesh distribution of triangular elements.

\begin{tabular}{lrl}
\hline Geometry & Nodes & Elements \\
\hline Rotating blades and nacelle & 47131 & 94178 \\
Stationary supporting hub & 5120 & 10160 \\
\hline
\end{tabular}

Table 2

Grid distribution and time step.

\begin{tabular}{|c|c|c|c|c|}
\hline Grid & & Nodes & $\begin{array}{l}\text { Grid size near } \\
\text { propeller }\end{array}$ & $\begin{array}{l}\text { Time steps per } \\
\text { rotation }\end{array}$ \\
\hline Grid A & 14 million & $201 \times 201 \times 351$ & $D / 83$ & 1000 \\
\hline Grid B & 47 million & $301 \times 301 \times 523$ & $D / 125$ & 2000 \\
\hline Grid C & 87 million & $377 \times 377 \times 611$ & $D / 192$ & 2000 \\
\hline Grid D & 179 million & $491 \times 491 \times 743$ & $D / 283$ & 3000 \\
\hline
\end{tabular}

along with generalized minimal residual method (GMRES) solver is used to solve the pressure Poisson equation. A matrix-free NewtonKrylov method is used for solving the discretized momentum equation. More details about the flow solver can be found in Kang et al. (2011), Yang et al. (2015) and Yang and Sotiropoulos (2018).

\section{Simulation details}

The VP1304 propeller shown in Fig. 1 is used for the present research, which was provided by SVA Potsdam in the SMP'11 workshop at https://www.sva-potsdam.de/en/pptc-smp11-workshop/. The VP1304 propeller is composed of five rotating blades with a central nacelle and a stationary supporting hub. The diameter of the propeller is $D=0.25 \mathrm{~m}$.

In the CURVIB method, the surfaces of the blades, nacelle and supporting hub are discretized using triangular meshes (with numbers of nodes and elements shown in Table 1), which are clustered around the tailing edge, leading edge and the root of the blades for better representation of the geometry as shown in Fig. 2(a). In immersed boundary methods, the background grid does not have to conform to the boundary of the immersed bodies. In this work, we adopt Cartesian grids with grid nodes uniformly distributed in the region around the propeller and gradually stretched to the far field as shown in Fig. 2(b). The size of the computational domain is $L_{x} \times L_{y} \times L_{z}=10 D \times 10 D \times 13.5 D$. The propeller is located $2.5 \mathrm{D}$ from the inlet and $11 \mathrm{D}$ from the outlet, respectively. Four sets of grids Grid A, Grid B, Grid C and Grid D with numbers of grid nodes 14 million, 47 million, 87 million and 179 million are employed, for which more details can be found in Table 2 . The incoming velocity is $U=7.204 \mathrm{~m} / \mathrm{s}$. The rate of rotor revolution is $n=-23 \mathrm{~s}^{-1}$, where the negative sign indicates rotating in reverse direction (negative $x$-direction as shown in Fig. 2(b)). The density of water is $997.1 \mathrm{~kg} / \mathrm{m}^{3}$. The kinematic viscosity of water at temperature $t_{w}=24.7^{\circ} \mathrm{C}$ is $0.903 \times 10^{-6} \mathrm{~m}^{2} / \mathrm{s}$. Given the above operational conditions, the advance ratio is $J_{A}=U /(n D)=-1.253$, and the Reynolds number is $R e=U D / v=2 \times 10^{6}$. The contours of the estimated $y^{+}$ of the grid cell near the propeller surface for the four sets of grids are illustrated in Fig. 3, where the estimation is based on local grid spacing $\Delta=J^{-1 / 3}$ and the Reynolds number defined using the relative incoming velocity at different radial locations. It can be seen that the maximum near wall $y^{+}$gradually decrease from about 2000 to about 200 as we refine the grid.

For each case, the simulation is first carried out until the flow is fully developed, and then flowfield is averaged for quantities of interest by continuing the simulation. For averaging flowfield, 77, 32, 30 and 25 rotor revolutions are used for Grid A, B, C and D, respectively. For one rotor revolution simulation, the wall-clock time is about 2, 6, 11, 29 hours for Grid A, B, C and D, which employ 240, 480, 480 and 640 compute nodes, respectively. To mitigate the negative effect of the relatively less time employed for temporal averaging especially
Table 3

The mean values and root mean square values of the thrust and side-force coefficients.

\begin{tabular}{lllll}
\hline & $\left\langle K_{T}\right\rangle$ & $K_{T}^{\text {r.m.s. }}$ & $\left\langle\left|K_{Y}\right|\right\rangle$ & $K_{Y}^{\text {r.m.s. }}$ \\
\hline Grid A & -0.755 & 0.0844 & 0.177 & 0.211 \\
Grid B & -0.745 & 0.0673 & 0.250 & 0.284 \\
Grid C & -0.853 & 0.0530 & 0.288 & 0.324 \\
Grid D & -0.847 & 0.0392 & 0.224 & 0.253 \\
\hline
\end{tabular}

for finer grids, we further average the time-averaged flow fields in the circumferential direction for computing mean flow quantities.

\section{Results}

In this section, we compare several quantities computed from LES of the VP1304 propeller for four different grids, which include force coefficients, instantaneous flow field, time-averaged and circumferentially averaged flow field, and phase-averaged flow field.

\subsection{Force coefficients}

First we compare the thrust coefficient $K_{T}$ and the side-force coefficient $K_{Y}$ predicted by the four different grids, which are defined by

$K_{T}=\frac{F_{x}}{\rho n^{2} D^{4}}, \quad K_{Y}=\frac{F_{y}}{\rho n^{2} D^{4}}$,

where $F_{x}$ and $F_{y}$ are the forces exerted on the blades in the axial and $y$-directions, respectively. As shown in left column of Fig. 4, the thrust coefficients computed from Grid C and Grid D, which are similar in magnitude, are smaller than that from Grid A and Grid B. The amplitudes of side-force coefficients computed from the four grids, on the other hand, are close to each other. It is also observed that the variation range of the thrust coefficient is smaller than that of the side-force coefficient. Detailed comparison of statistics of $K_{T}$ and $K_{Y}$ computed from different grids is shown in Table 3.

In the right column of Fig. 4, we compare the pre-multiplied power spectral densities (PSD) of $K_{T}$ and $K_{Y}$ computed from the four different grids. On the right of Fig. 4(a), two peaks are observed at $f_{0}$, and $4 f_{0}$ for the pre-multiplied PSD of $K_{T}$, where $f_{0}$ is the blade passing frequency (BPF). On the right of Fig. 4(b), peaks of the pre-multiplied PSD of $K_{Y}$ are observed at $f_{0}, 3 f_{0}$ and $5 f_{0}$, respectively. As the direction of the side force is fixed, the peaks of BPF and its multipliers appear in its premultiplied PSD as a result of propeller rotation. For the peaks observed on PSD of $K_{T}$, we suspect that they are caused by the asymmetrical oncoming flow induced by propeller blades. It is noticed on the right of Fig. 4(a) that the magnitudes of these peaks are reduced as we refine the grid. It will be shown in Appendix that it is caused by the reduced degree of asymmetry when refining the grid.

Measurements of this propeller in crashback mode do not exist for neither force coefficient nor wake velocities. We attempt to evaluate the present simulation results by comparing the force coefficients computed in this work with the experimental results (Chang et al., 2008b; Jessup et al., 2004) of the propeller 4381. As seen in Fig. 5(a), the thrust coefficients computed in this work are similar to those of the 4381 propeller. The magnitudes of the side-force coefficients of this propeller, on the other hand, are larger than those of the 4381 propeller as shown in Fig. 5(b).

\subsection{Instantaneous flow field}

Here, we show the instantaneous streamwise velocity at the central slice $z=0$ in Fig. 6 computed from the four grids. As seen the flow is highly unsteady with massive flow separation and reverse flow region, which exists for $y / D<1$, while slightly accelerated for $y / D>1$. The 


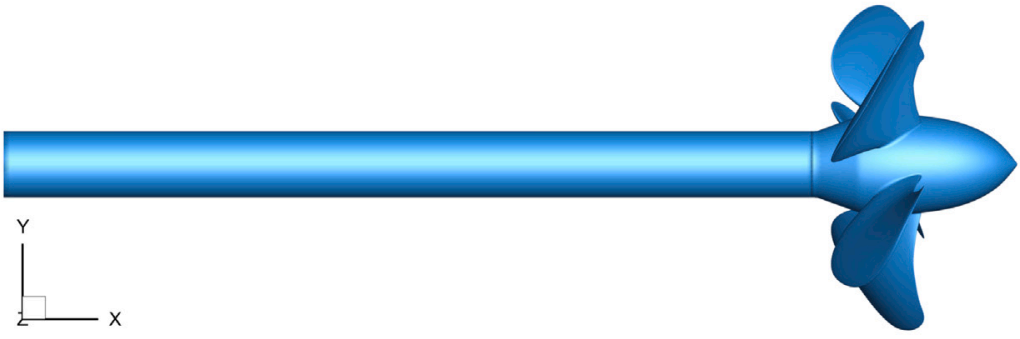

(a) Side view

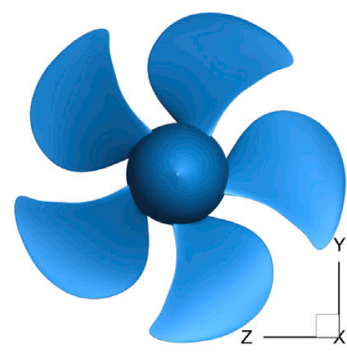

(b) Front view

Fig. 1. Geometry of the VP1304 propeller. The flow is in the positive $x$-direction.

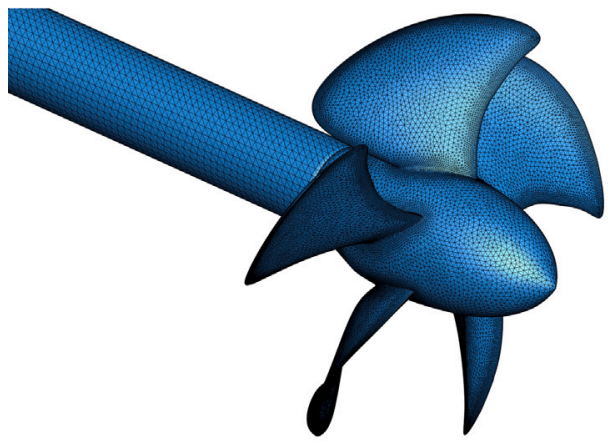

(a) Triangular surface mesh representing the geometry of the propeller.

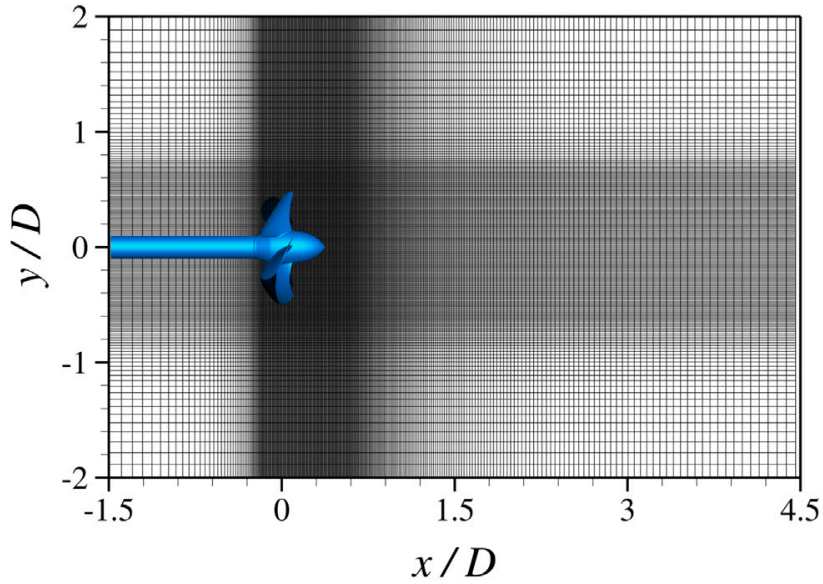

(b) Background Cartesian grid for solving fluid flows.

Fig. 2. (a) Surface mesh and (b) background Cartesian grid for solving the flow over the VP1304 propeller using large-eddy simulation with the CURVIB method.

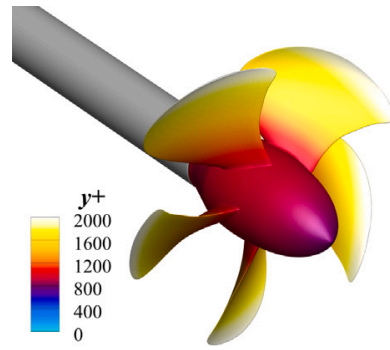

(a) Grid A

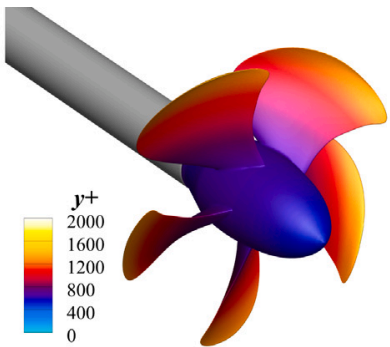

(b) Grid B

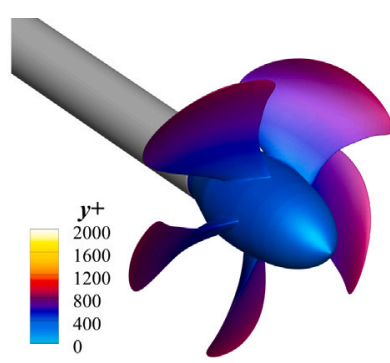

(c) Grid C

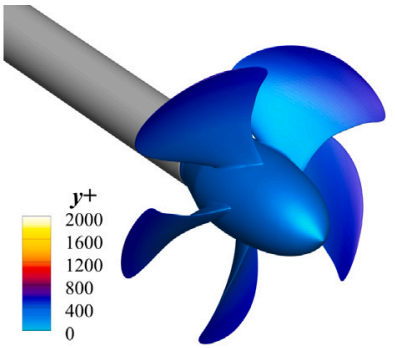

(d) Grid D

Fig. 3. Estimated $y^{+}$of the grid cell near the propeller surface for Grid A, B, C and D.

reverse flow region ends at about $x / D=1.5$, and starts recovering at further downstream locations. Overall the flow patterns downstream of the propeller are very similar for different grids, except for that the length of reverse flow region seems to be longer for Grid D while shorter for Grid A. Then, we compare the vortex structures identified using the Q-criterion $(Q=500)$ computed with the four grids in Fig. 7. It can be observed that fine vortex structures are observed from the Grid D case. Other than this, no significant differences are observed among four different grids.

\subsection{Averaged flow field}

In this section, we compare the temporally and circumferentially averaged flow fields computed from the four grids, which is denoted by $\langle\cdot\rangle$. First we compare the averaged streamlines at the central slice $z=0$ in Fig. 8. As seen the length and width of the recirculation bubble
Table 4

Comparison of the length and width of the separation bubble computed from different grids.

\begin{tabular}{lllll}
\hline & Grid A & Grid B & Grid C & Grid D \\
\hline Length & $1.55 D$ & $1.63 D$ & $1.68 D$ & 1.905 \\
Width & $1.64 D$ & $1.64 D$ & $1.62 D$ & $1.60 D$ \\
\hline
\end{tabular}

are approximately $1.5 D \sim 2.0 D$ and $1.6 D$, respectively. Compared with those simulated using Grid A, B and C, the length of the recirculation bubble from Grid D is slightly longer. Detailed comparison of the length and width of the recirculation bubble is shown in Table 4.

Then, we show the averaged streamwise velocity $\langle u\rangle / U$ at slice $z=0$ in Fig. 9. As seen the four grids produce similar flow patterns, except that the reverse flow region from Grid D is longest among the four grids. For LES on a very coarse grid, a considerable part of the energetic 

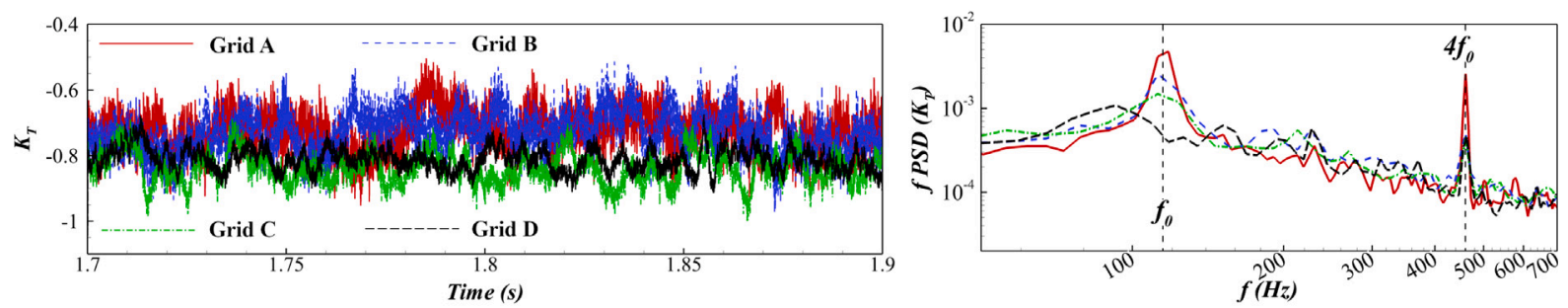

(a) Thrust coefficient
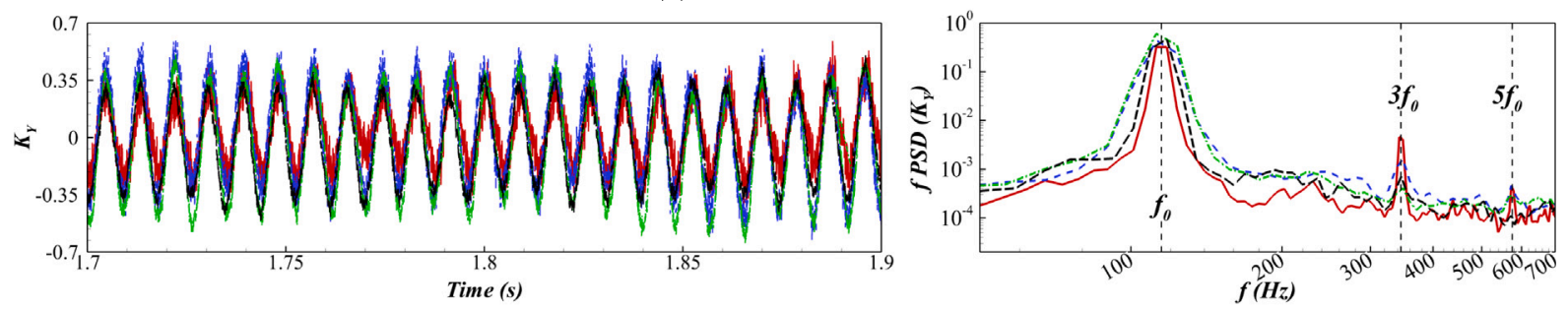

(b) Side-force coefficient

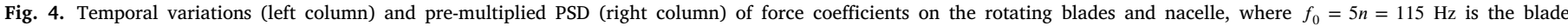
passing frequency.

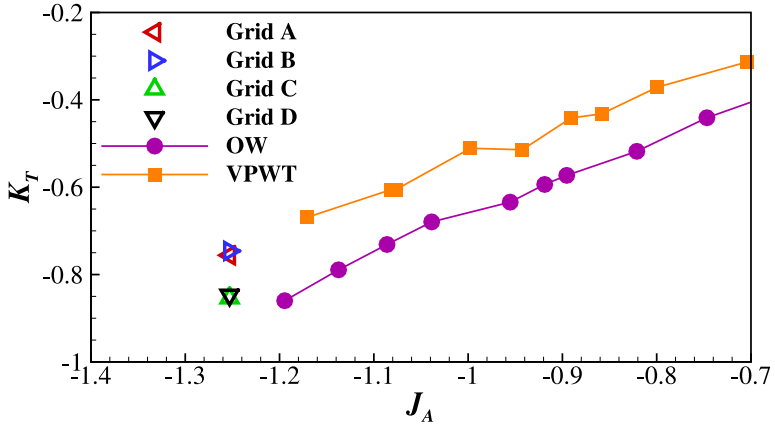

(a) Mean thrust coefficient $\left\langle K_{T}\right\rangle$.

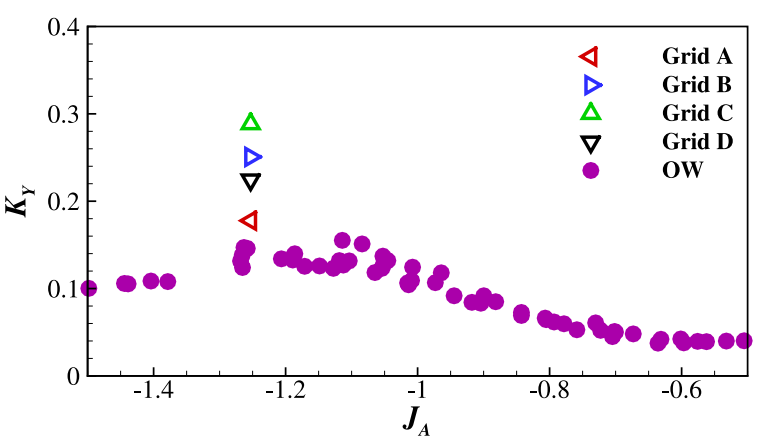

(b) Mean side-force coeffcients magnitude $\left\langle\left|K_{Y}\right|\right\rangle$.

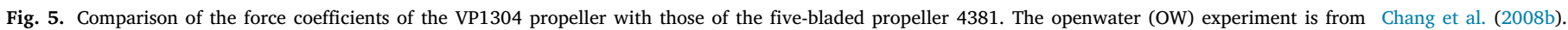
The variable pressure water tunnel (VPWT) result is from Jessup et al. (2004).

eddies is modeled by the SGS model, which essentially increases the dissipation of the flow and thus accelerates the recovery of the propeller wake. This explains why the wake computed using Grid D propagates further in the downstream of the propeller. Moreover, similar trend was also observed in the grid refinement study on the flow past a circular cylinder (Rosenfeld, 1994). To make quantitative comparison, the averaged streamwise velocity profiles at several downstream locations are shown in Fig. 10. Noticeable differences can be observed in the near wake region at $x / D=0.6$, which probably result from the differences in grid resolutions near the blades. The discrepancies among the four grids are relatively large at $x / D=1.4$, where the separation bubble ends in the vicinity (which is relatively sensitive to the grid resolution). At further downstream locations where $x / D>2.4$, the discrepancies among the four grids become small.

Further comparison among the four grids is made for the turbulence kinetic energy (TKE) as shown in Fig. 11. As seen significant differences exist in the TKE of the four grids that the grid with higher resolution produces lower TKE, which indicates that sub-grid scale dissipation might not be enough for the coarse grid. For quantitative comparison, the TKE profiles at several downstream locations are shown in Fig. 12. It can be observed that the discrepancies among the four grids are significant in the near wake locations where $x / D<2.4$, which is probably caused by different grid resolutions. At far wake locations where $x / D \geq 4.0$, the differences in the TKE profiles among the four grids are significantly reduced, which indicates the far wake TKE is less sensitive to grid resolution in the near propeller region. Another observation in the profiles of TKE is that the wake gradually expands in the radial direction along the streamwise direction, which becomes slower as we refine the grid.

\subsection{Phase-averaged flow field}

In this section, we compare the phase-averaged flow fields computed from the four grids. Different from Section 4.3, the ensemble average $\langle\cdot\rangle$ indicates phase average in this section. Since the VP1304 propeller has five blades, five phases in each rotation are used for the averaging.

We first show the phase-averaged Q-criterion iso-surfaces computed from the four grids in Fig. 13. Clear tip vortices are observed for all grids with those from Grid A and B smoother than Grid C and D. For Grid D, the tip vortices are observed having finer structures and longer tails as compared with the other three grids. Because of the highly turbulent flow in the immediate downstream of the propeller, such organized coherent structures along the tip does not persist to further downstream locations as often observed in the forward mode.

As illustrated in Fig. 14, we further show phase-averaged flow fields at different radial locations, i.e. $r / R=0.4, r / R=0.6, r / R=0.8$ and $r / R=1.0$ where the radial location $r$ is defined as $r=\sqrt{y^{2}+z^{2}}$ (the rotor is located at $(0,0,0)$ ), and $R=D / 2$ is the radius of the 


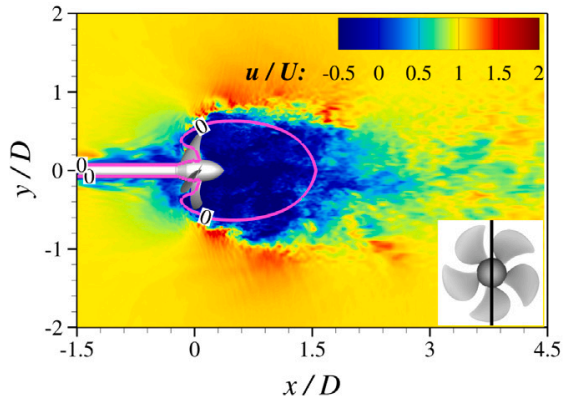

(a) Grid A

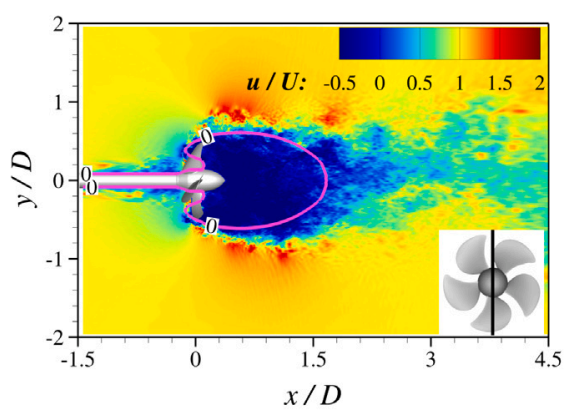

(c) Grid C

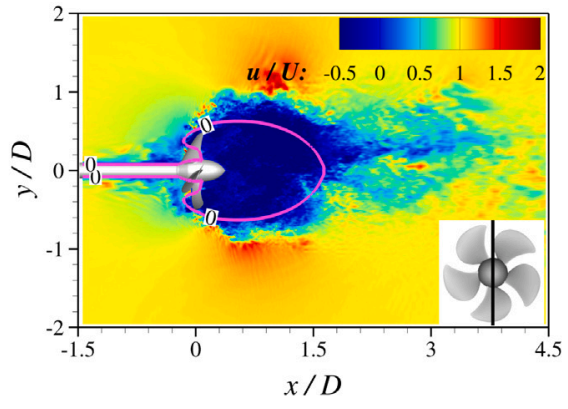

(b) Grid B

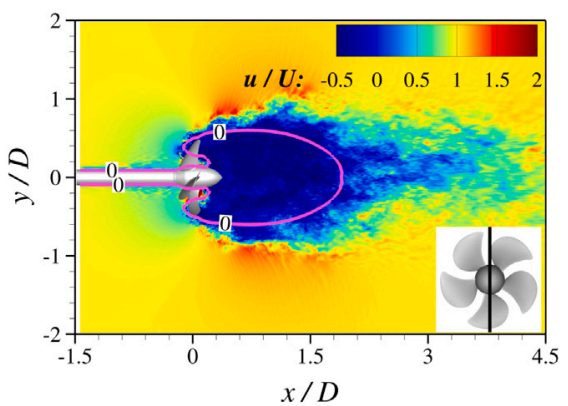

(d) Grid D

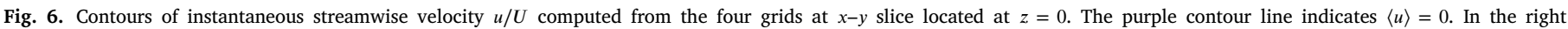
bottom of each subfigure, the rotation phase of the propeller is shown.

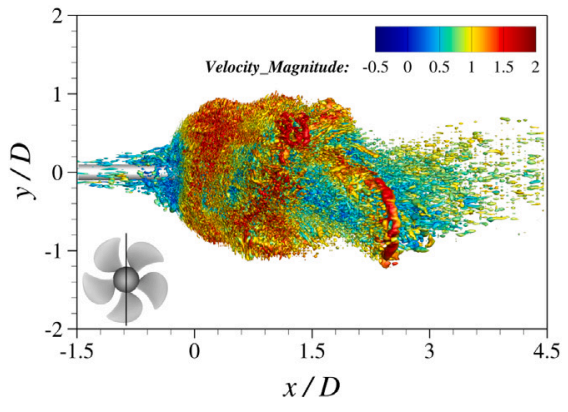

(a) Grid A

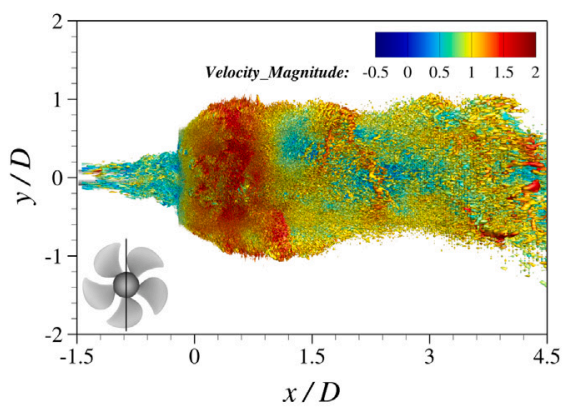

(c) Grid C

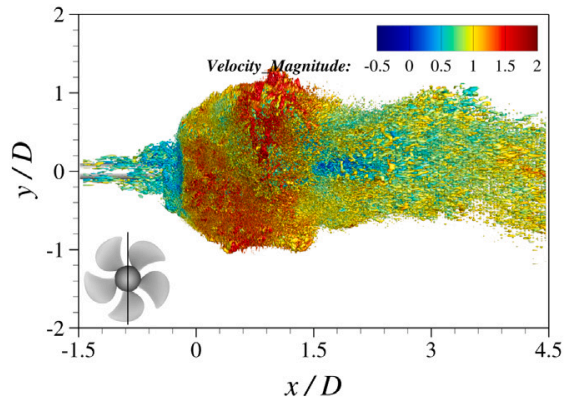

(b) Grid B

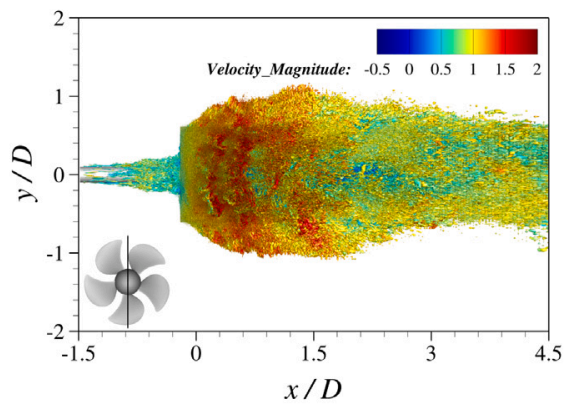

(d) Grid D

Fig. 7. Instantaneous Q-criterion iso-surfaces computed from the four grids with $Q=500$. In the left bottom of each subfigure, the rotation phase of the propeller is shown.

propeller. The phase-averaged streamlines on the slices at different radial locations are shown in Fig. 15, where the coordinates are defined as $(x / D, \theta)$, and the velocity components for drawing streamlines are defined as $(\langle u\rangle / D,\langle\omega\rangle)$. It can be found that the four grids produce similar overall streamline patterns at each radial location with the longest reverse flow region observed in the Grid D case, which is consistent with observations in Fig. 9. The azimuthal velocity in the wake is observed in the positive $\theta$ direction induced by the blade rotation. At radial locations $r / R=0.4$ and $r / R=0.6$, the streamlines near the tailing-edge of the blades computed from Grid $C$ and Grid D are quite different from those from Grid A and Grid B, which become close to each other as moving outward in the radial direction. Additionally, 


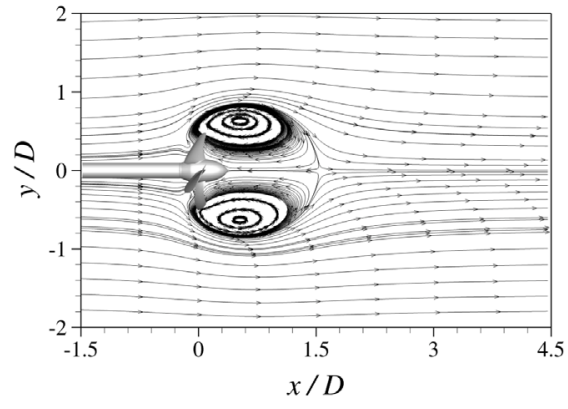

(a) Grid A

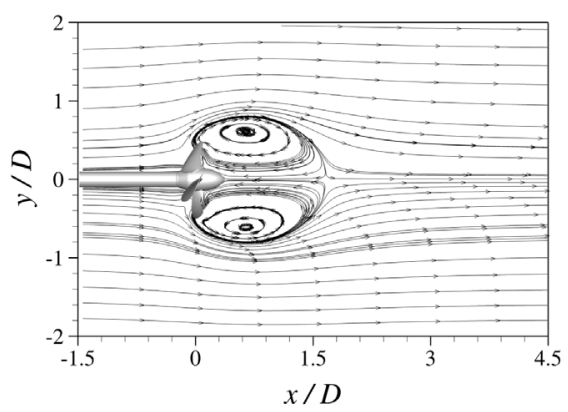

(c) Grid C

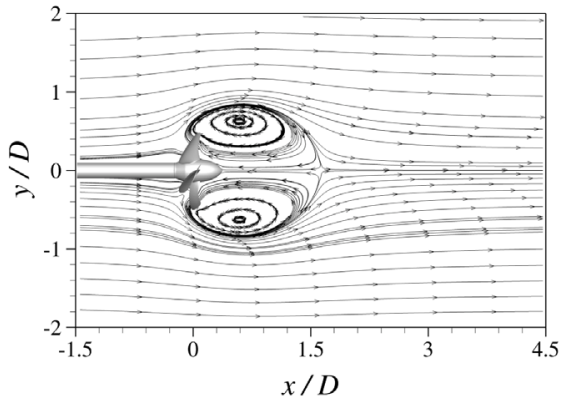

(b) Grid B

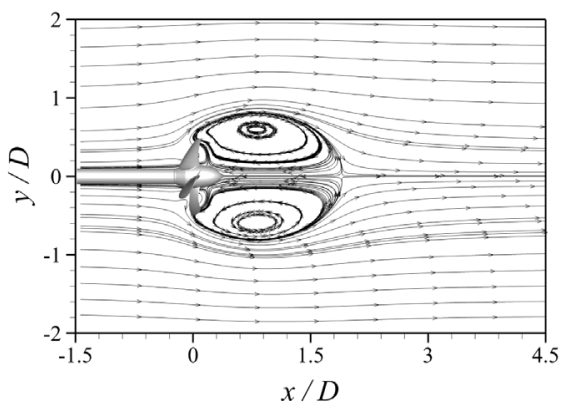

(d) Grid D

Fig. 8. Temporally and circumferentially averaged streamline computed from four grids at $x-y$ slice located at $z=0$.

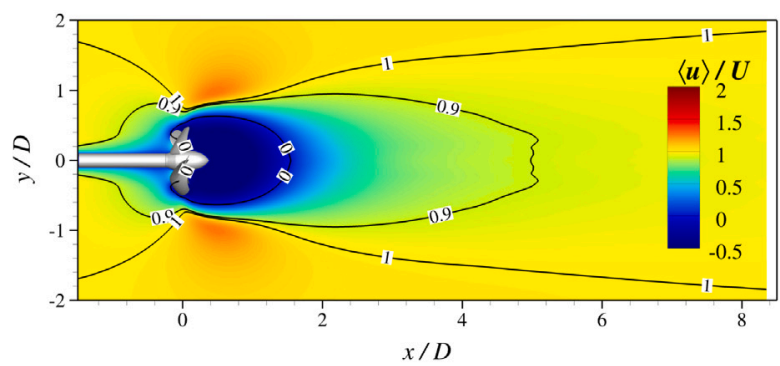

(a) Grid A

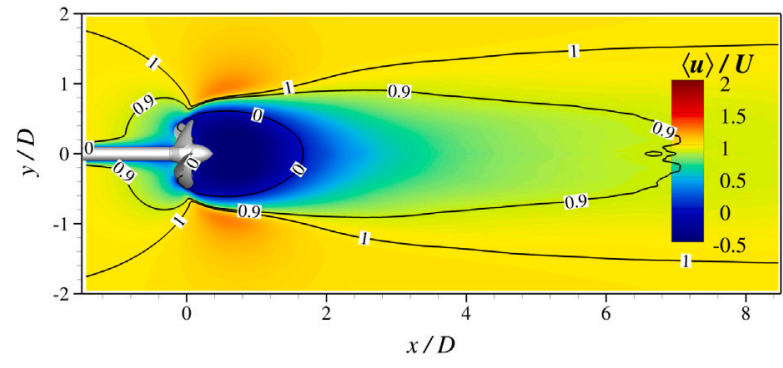

(c) Grid C

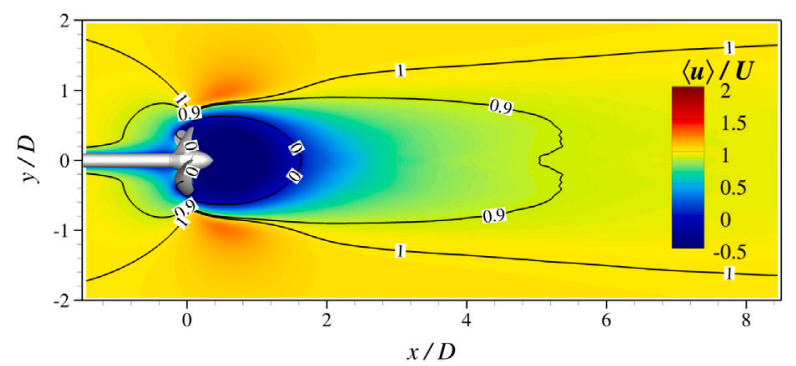

(b) Grid B

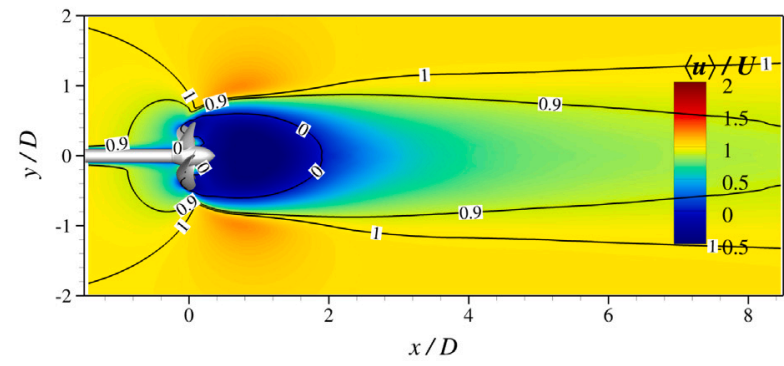

(d) Grid D

Fig. 9. Temporally and circumferentially averaged streamwise velocity $\langle u\rangle / U$ at $x-y$ slice located at $z=0$.

it is observed that the thickness of the foil section (which is represented by the grid cells located in the foil and the grid cells in the fluid with at least one neighbor located in the solid) from Grid A case is noticeably thicker than those from Grid B, C and D cases.

We then examine the phase-averaged streamwise velocity $\langle u\rangle / U$ computed from four grids in Fig. 16. The differences among the four grids are significant at $r / R=0.4$, where the grid with higher spatial resolution produces significantly larger streamwise velocity. Such strong jet-like flows between two adjacent blades are related to highly threedimensional flow structures near the root of the blades. At $r / R=0.6$ and $r / R=0.8$, on the other hand, the phase-averaged streamwise velocity $\langle u\rangle / U$ in the downstream of the blades are dominated by reverse flows caused by massive flow separations at corresponding radial locations. At the radial location $r / R=1.0$, the streamwise velocity is not significantly decelerated, because of the effect of blades' tip loss.

Following the streamwise velocity, we here examine the phaseaveraged the radial velocity in Fig. 17, which is obtained through decomposition of velocity into the radial, tangential and streamwise components, with the radial direction and tangential direction located 


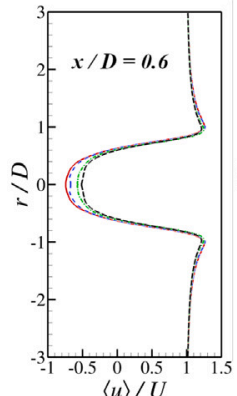

$\langle u\rangle / U$

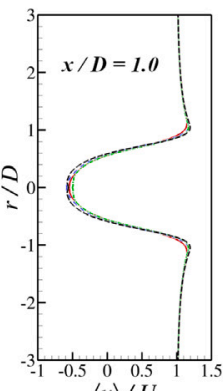

$\langle u\rangle / U$

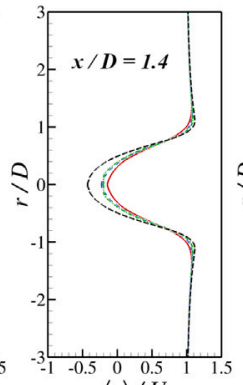

$\langle u\rangle / U$

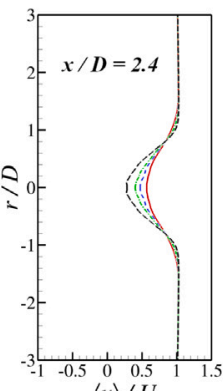

$\langle u\rangle / U$

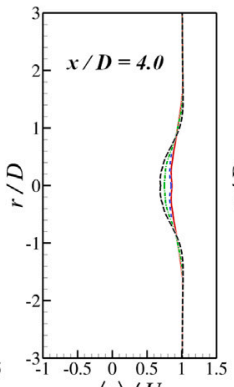

$\langle u\rangle / U$

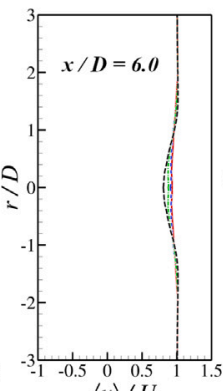

$\langle u\rangle / U$

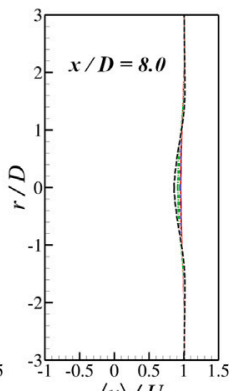

$\langle u\rangle / U$

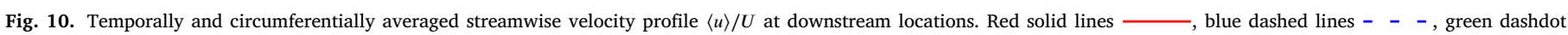
lines -... and black dashed lines - - - represent results of Grid A, B, C and D, respectively.

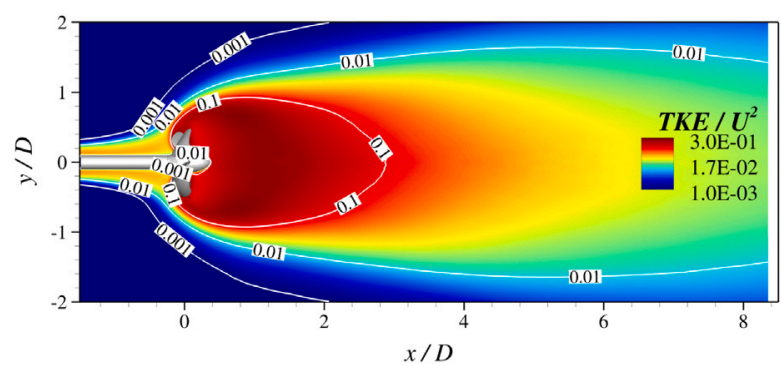

(a) Grid A

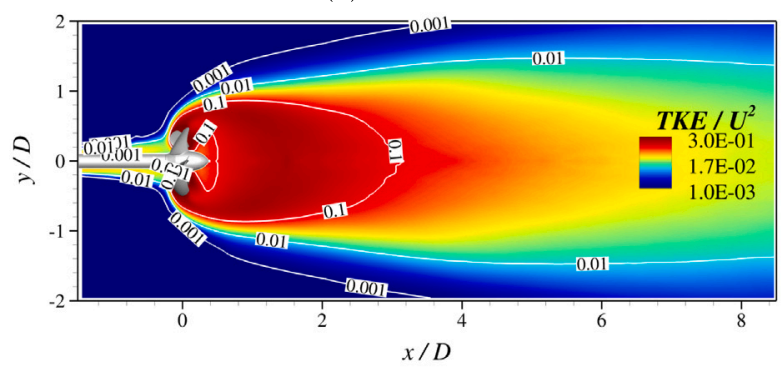

(c) Grid C

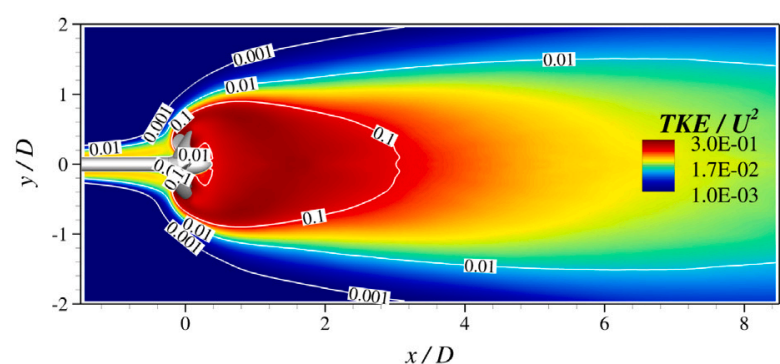

(b) Grid B

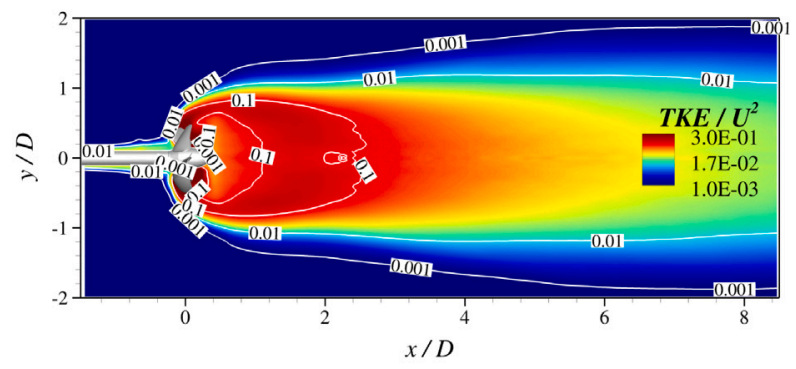

(d) Grid D

Fig. 11. Circumferentially averaged $\mathrm{TKE} / U^{2}$ at $x-y$ slice located at $z=0$.
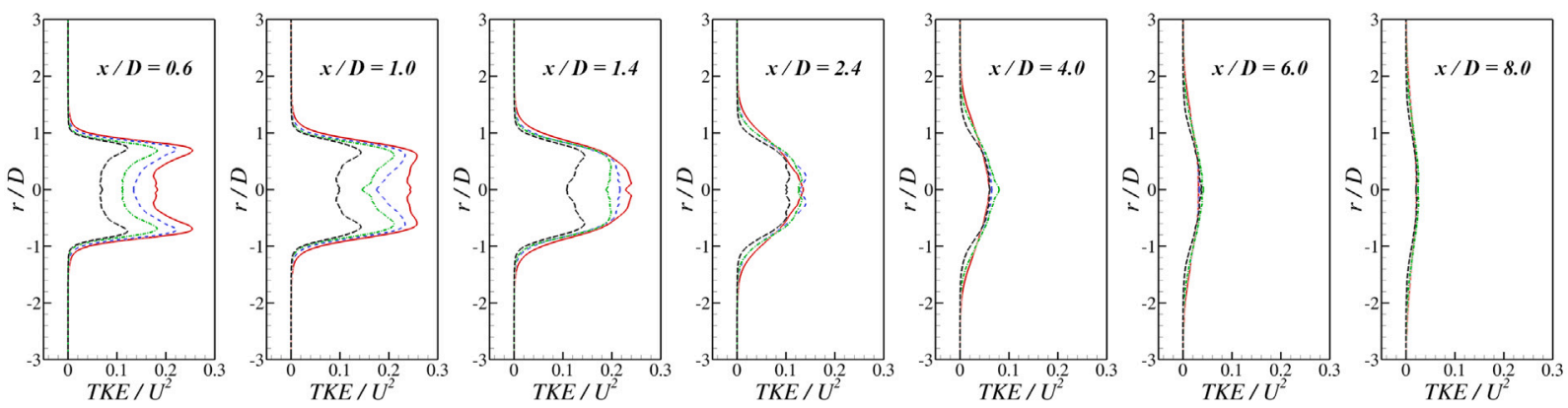

Fig. 12. Circumferentially averaged turbulence kinetic energy TKE $/ U^{2}$ at different downstream locations. Red solid lines ...... and black dashed lines - . - - represent results of Grid A, B, C and D, respectively.

in the $y-z$ plane (rotor rotating plane). It can be seen that the flow patterns identified using the radial velocity are different at different radial locations that the inward (negative radial velocity) motion and outward motion (positive radial velocity) are observed near the edge facing the undisturbed flow and the wake, respectively at $r / R=0.4,0.6$, which are reversed at $r / R=0.8,1.0$ locations. Similar to the observation from the streamwise velocity, the differences between the results from different grids are more significant at locations close to root and less significant at locations close to the tip.
Here we show the phase-averaged tangential velocity in Fig. 18, which is defined as positive for the rotating speed in the " $-x$ "-direction. It is found that the tangential velocity has higher magnitudes at the radial locations $r / R=0.6$ and $r / R=0.8$ as compared to those at $r / R=0.4$ and $r / R=1.0$. The tangential velocity is observed to increase as the grid is refined especially at locations $r / R=0.4,0.6$ and 0.8 .

At last we compare the phase-averaged turbulence kinetic energy TKE $/ U^{2}$ in Fig. 19. It is observed that at $r / R=0.4$ and $r / R=0.6$, the TKE computed using Grid D is significantly highest among the four 

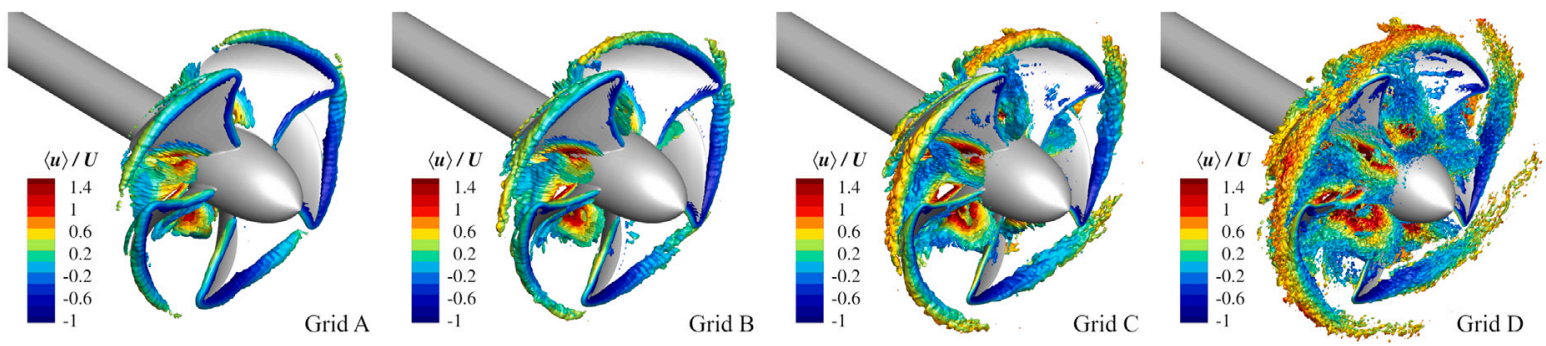

Fig. 13. Phase-averaged Q-criterion iso-surfaces computed from the four grids.

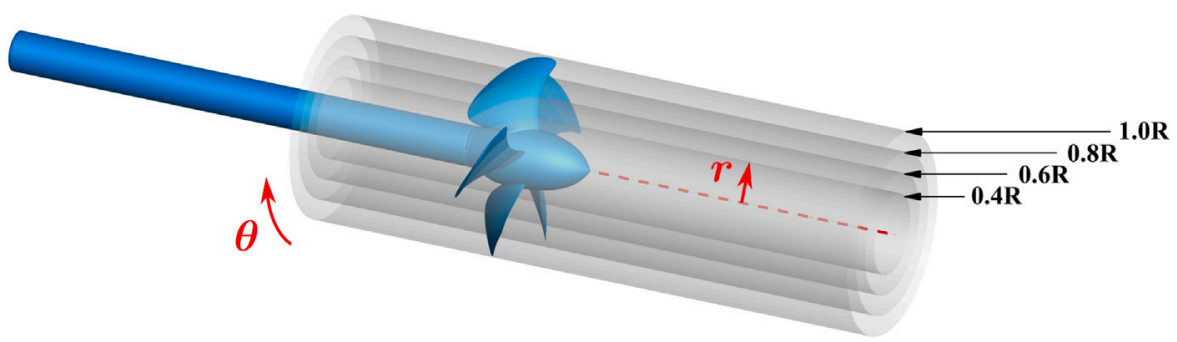

Fig. 14. Illustration for the slices at different radial locations for the phase-averaged results.
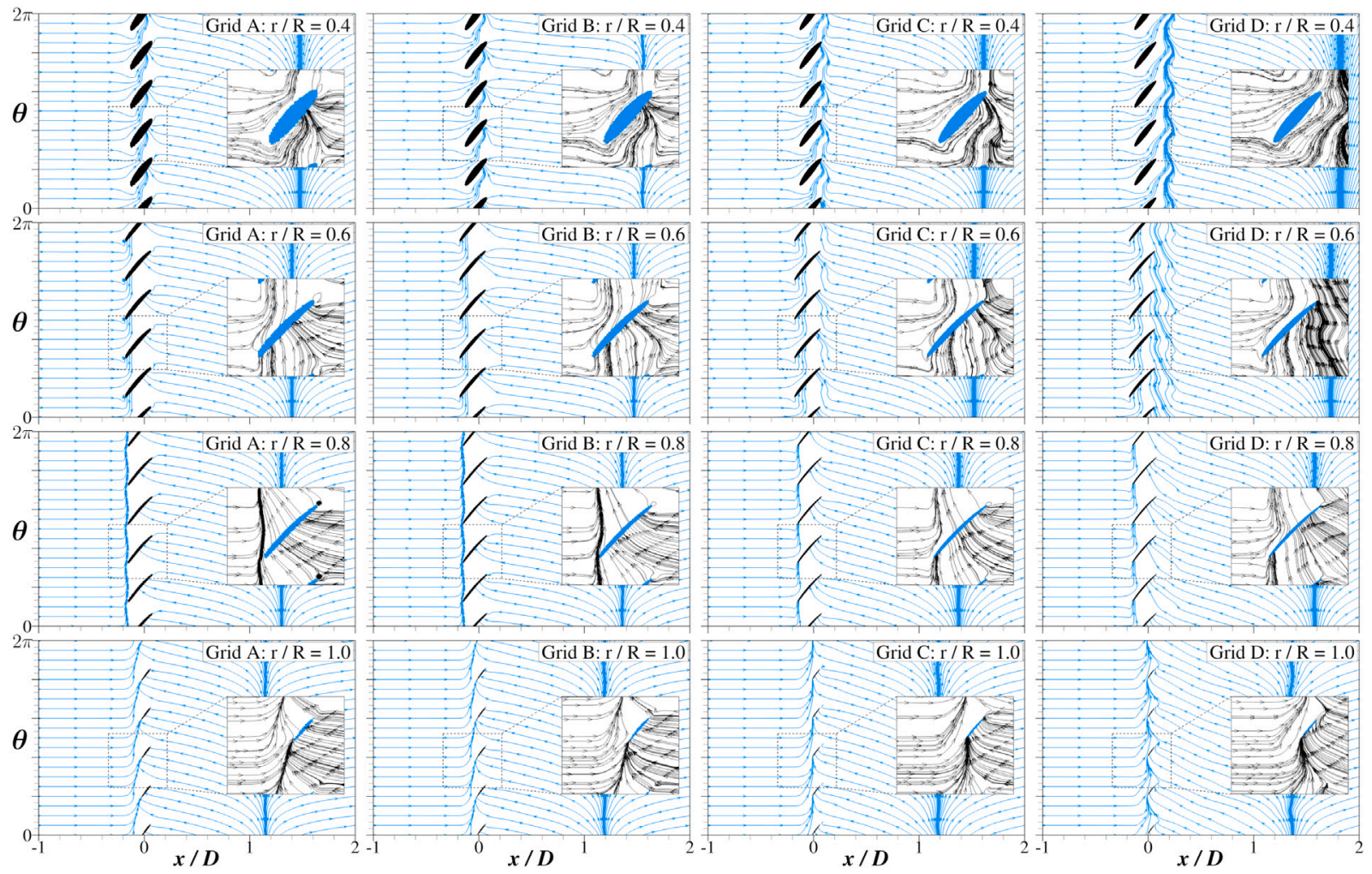

Fig. 15. Phase-averaged streamlines at different radial slices. The velocity for drawing streamline is defined in the inertial frame of reference as $(\langle u\rangle / D,\langle\omega\rangle)$.

grids in the region around the blades. Furthermore, it is observed that the patterns of high TKE region are also different between grids of different resolutions. In the immediate downstream of the blades, a region of high TKE is observed at $r / R=0.4,0.6$ in the case using Grid $\mathrm{D}$, which does not exist for Grid A and is under-predicted for Grids B and C. In the wake of the propeller $(x / D>0.4)$, on the other hand, the TKE computed using Grid D is lower than those computed from other three grids.

\subsection{Summary of errors evaluated for different flow quantities}

To quantitatively evaluate the grid-dependence of different quantities, we compute the errors of different quantities, i.e. the thrust coefficient, and the velocity and TKE at different downstream locations.

The relative error of the thrust coefficient is defined as

$\operatorname{Error}\left(K_{T}\right)=\left|\frac{{\overline{K_{T}}}-{\overline{K_{T}}}^{\mathrm{ref}}}{{\overline{K_{T}}}^{\mathrm{ref}}}\right| \times 100 \%$, 


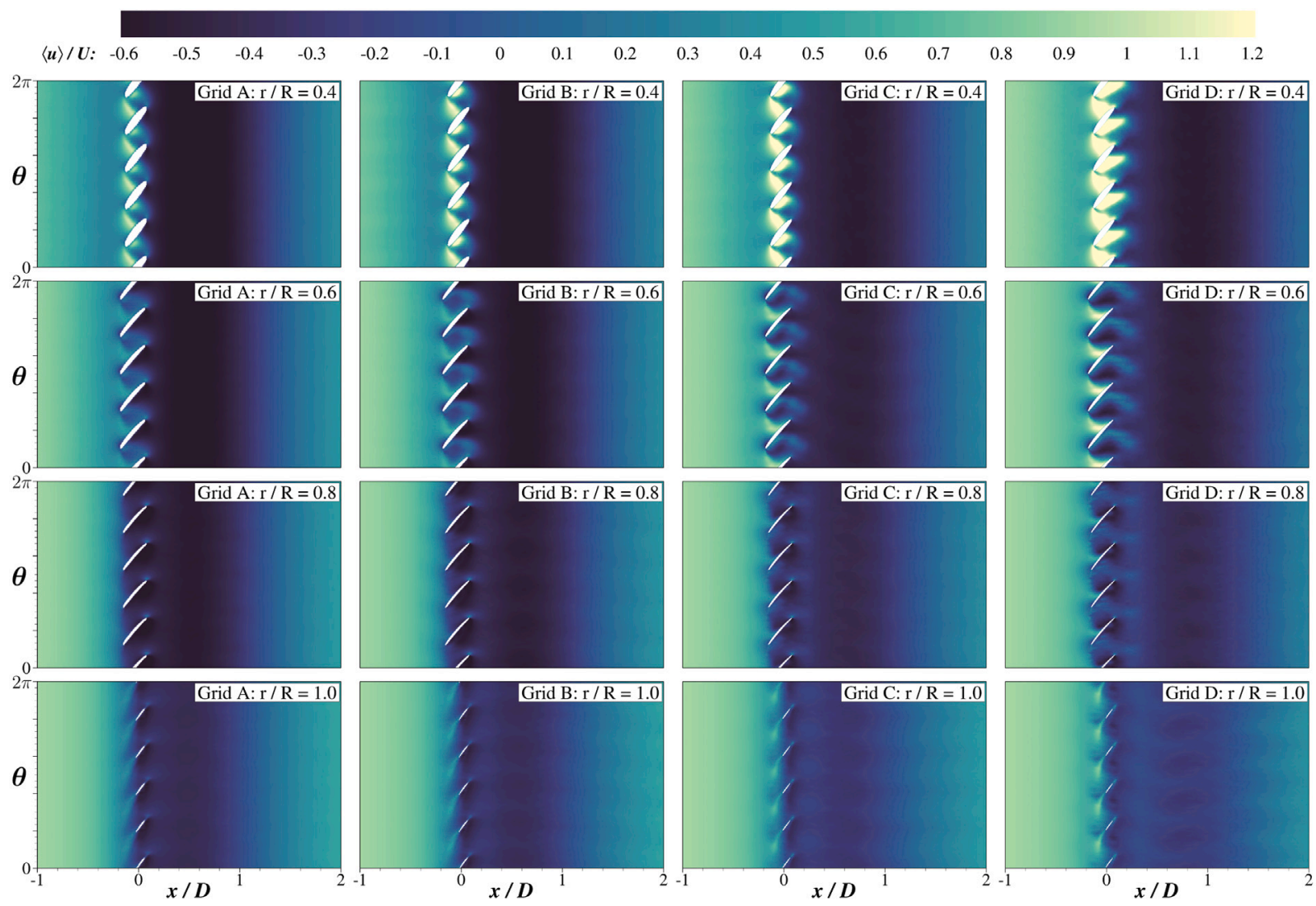

Fig. 16. Contours of phase-averaged streamwise velocity $\langle u\rangle / U$ at different radial slices.

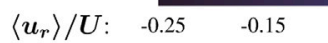

\section{$-0.0$}
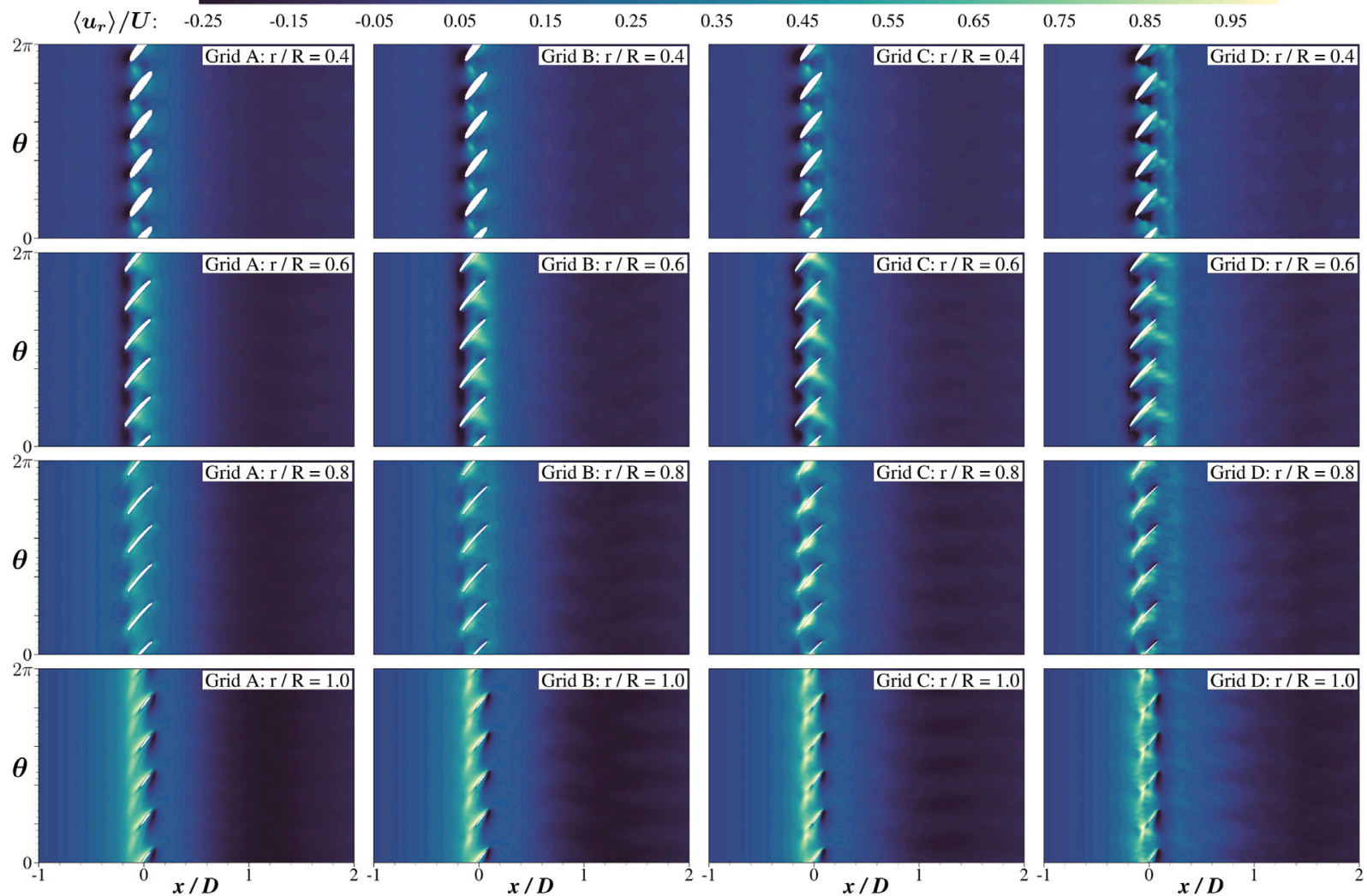

Fig. 17. Contours of phase-averaged radial velocity $\left\langle u_{r}\right\rangle / U$ at different radial slices. 


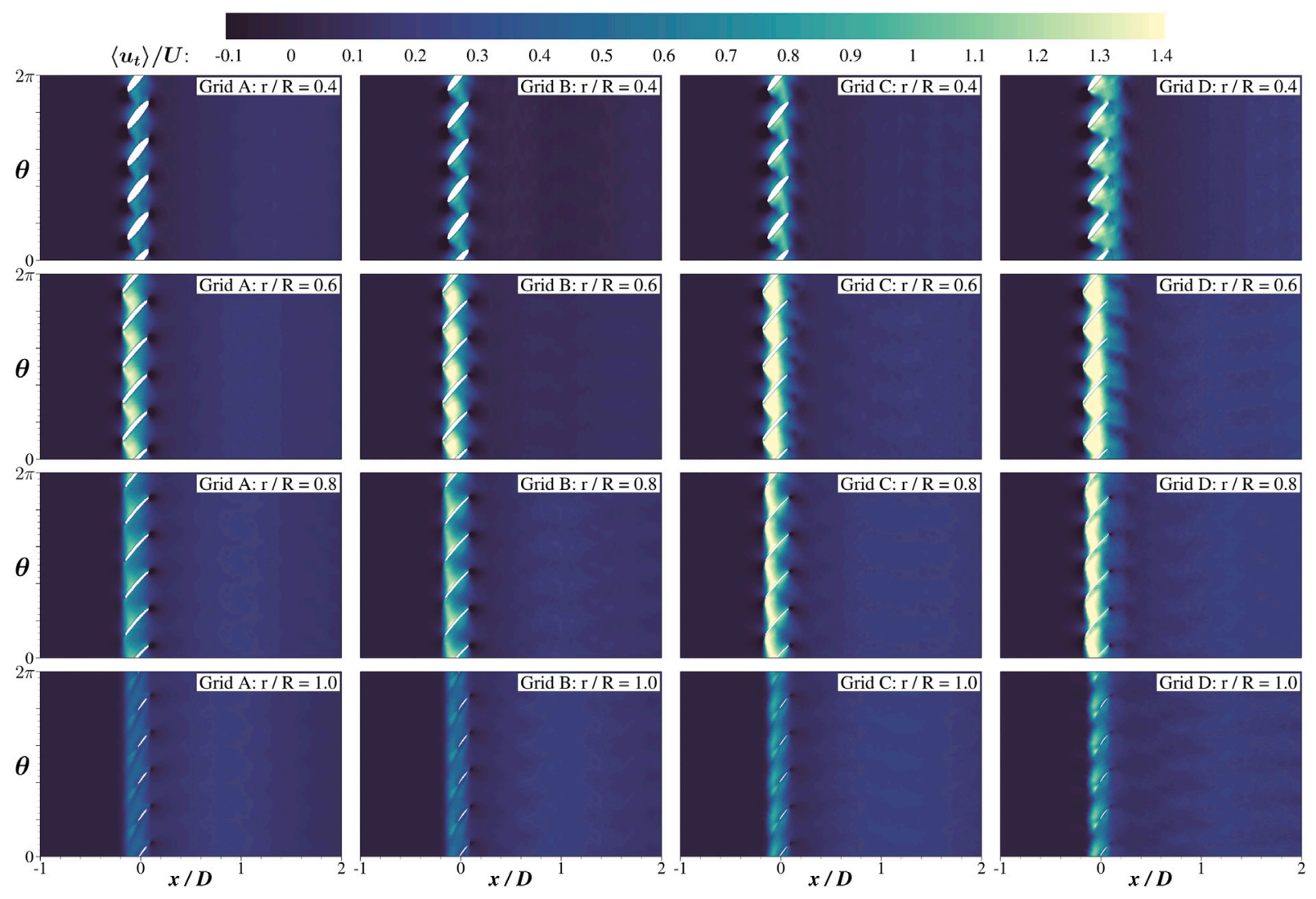

Fig. 18. Contours of phase-averaged tangential velocity $\left\langle u_{t}\right\rangle / U$ at different radial slices.

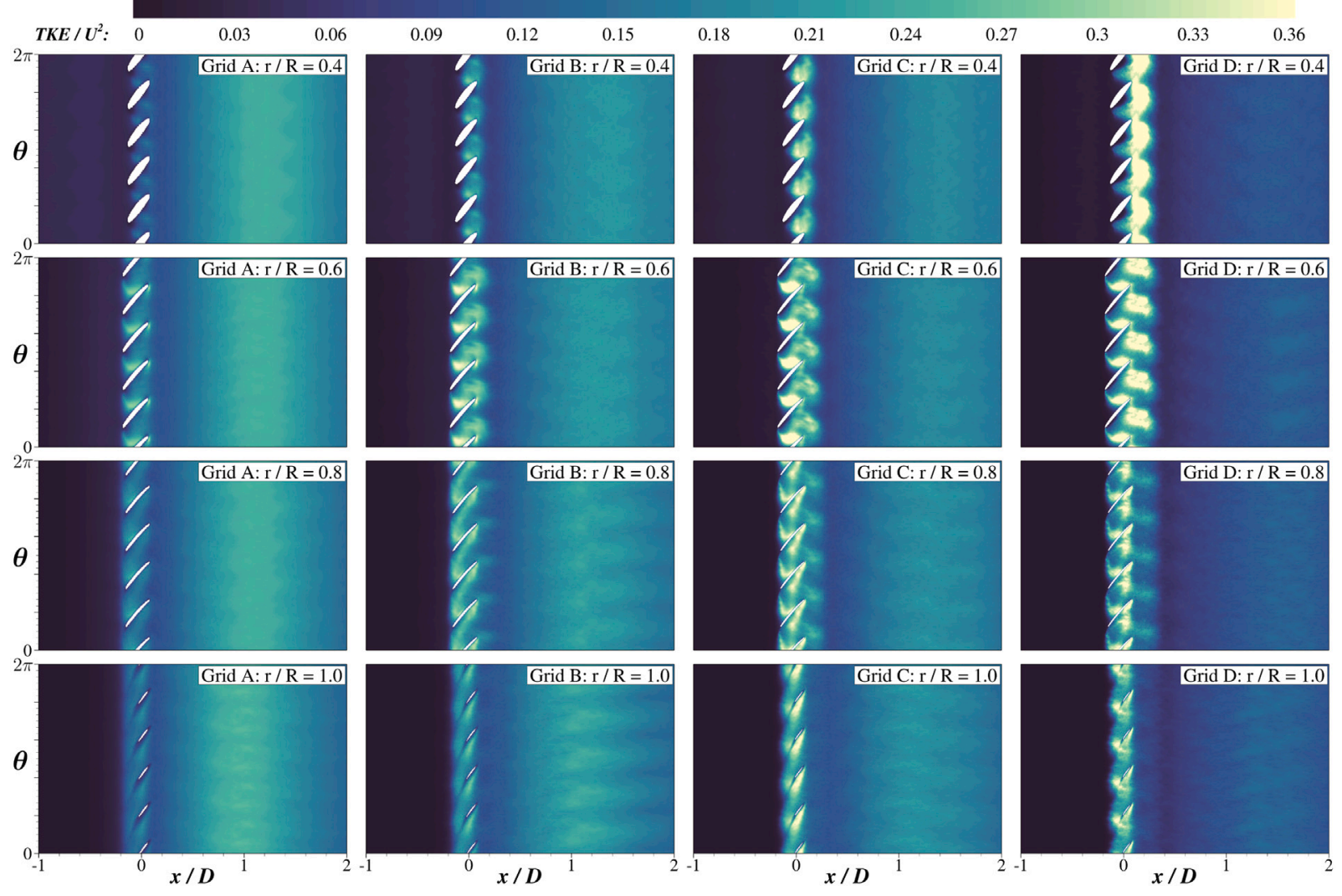

Fig. 19. Contours of phase-averaged turbulence kinetic energy TKE $/ U^{2}$ at different radial slices. 


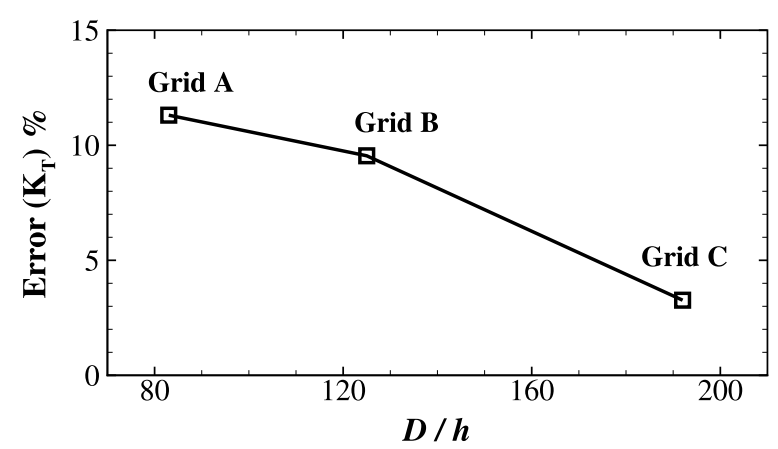

Fig. 20. Relative errors for $\overline{K_{T}}$.

where the overline indicates temporally averaged value, and the reference $\overline{K_{T}}$ ref is predicted by Grid D. The obtained relative error of $\overline{K_{T}}$ is shown in Fig. 20, where the horizontal axis $D / h$ indicates the number of cells distributed in the diameter of the propeller and $h$ represents the cell size. As seen, the relative error of thrust coefficient is reduced as the increase of the number of grid cells.

The normalized errors for the temporally, radially and circumferentially averaged streamwise velocity and turbulence kinetic energy are defined as follows:

$$
\begin{gathered}
\operatorname{Error}(\langle u\rangle)=\frac{1}{U} \frac{1}{N_{r} N_{c}} \sum_{k=1}^{N_{c}} \sum_{j=1}^{N_{r}}\left(\langle u\rangle_{j, k}-\langle u\rangle_{j, k}^{\mathrm{ref}}\right), \\
\text { Error(TKE) }=\frac{1}{U^{2}} \frac{1}{N_{r} N_{c}} \sum_{k=1}^{N_{c}} \sum_{j=1}^{N_{r}}\left(\mathrm{TKE}_{j, k}-\mathrm{TKE}_{j, k}^{\mathrm{ref}}\right),
\end{gathered}
$$

where $N_{c}$ and $N_{r}$ are the numbers of cells in the circumferential and radial directions, respectively. The reference values of $\langle u\rangle^{\text {ref }}$ and TKE ${ }^{\text {ref }}$ are from the Grid D case. As seen in Fig. 21(a), the error of the streamwise velocity gradually reduces as refining the grid for different downstream locations. As for the error of TKE shown in Fig. 21(b), it is observed that the error decreases as refining the grids for $x / D<2$, whereas at further downstream locations the errors from different grids are similar with each other. The error of the time-averaged streamwise velocity and the TKE along the centerline of the propeller is also examined. As shown in Fig. 22, trends similar with Fig. 21 are observed.

\section{Conclusions}

Large-eddy simulation of the propeller VP1304 in crashback mode is carried out at advance ratio $J_{A}=-1.253$ using the curvilinear immersed boundary method with four gradually refined grids of number of grid nodes 14, 47, 87 and 179 million. Quantities including force coefficients, time-averaged streamlines, velocities and TKE, and phase-averaged streamlines, velocities and TKE have been calculated to evaluate the grid dependence of different flow characteristics. Several conclusions can be drawn as follows: (1) the thrust coefficient is relatively more sensitive to the grid resolution compared with side-force coefficient; (2) in the near wake $(x / D \leq 2.4)$ the vortical structures shedding from the blades, the length of the reverse flow region, and the TKE are sensitive to grid resolution, while the time-averaged streamwise velocity are not sensitive to grid resolution; (3) in the far wake $(x / D \geq 4.0)$ the differences among the predictions from different grids are relatively small; (4) for the flow around the blades significant differences are observed for both averaged velocity field and the intensity and distribution of TKE at locations in the middle and close to root of the blade.

As shown in this work and other work in the literature (Vysohlid and Mahesh, 2006, 2007; Chang et al., 2008a; Verma et al., 2012; Jang and Mahesh, 2013; Kumar and Mahesh, 2016), the flow in the near wake of a crashback propeller is featured by complex turbulent

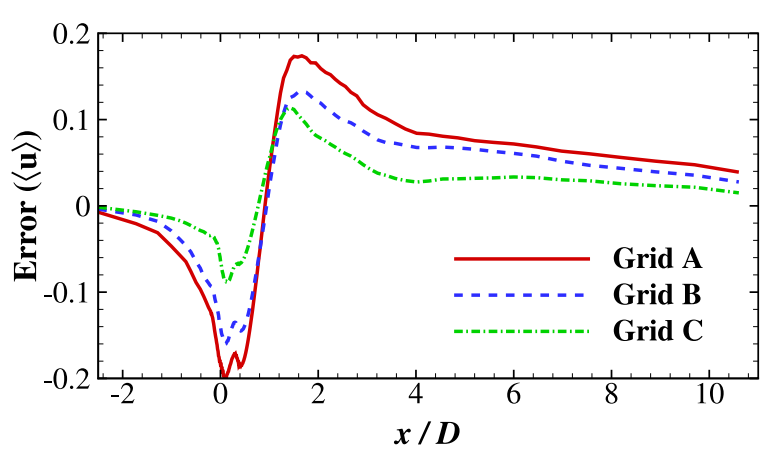

(a) $\operatorname{Error}(\langle u\rangle)$

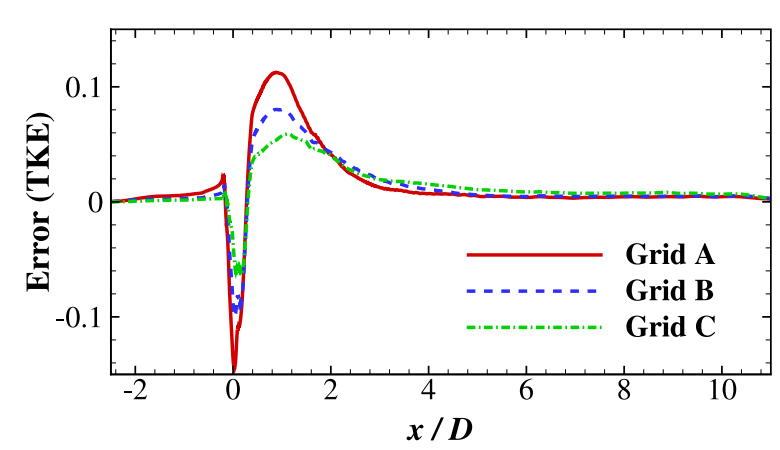

(b) Error(TKE)

Fig. 21. Errors of temporally, radially and circumferentially averaged streamwise velocity and TKE along the streamwise direction.

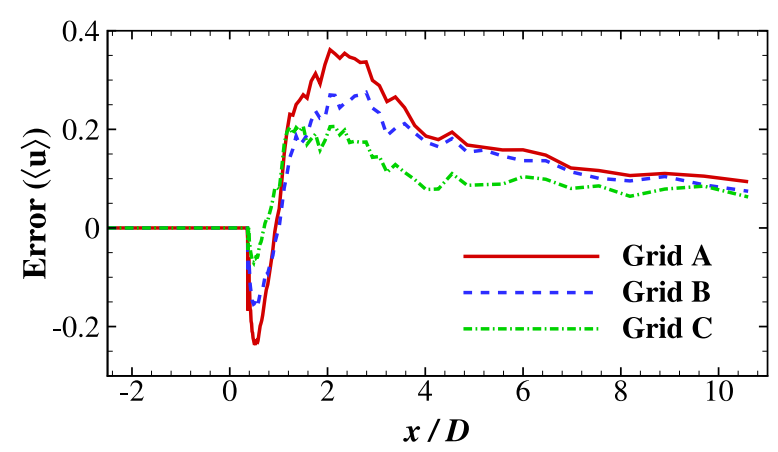

(a) $\operatorname{Error}(\langle u\rangle)$

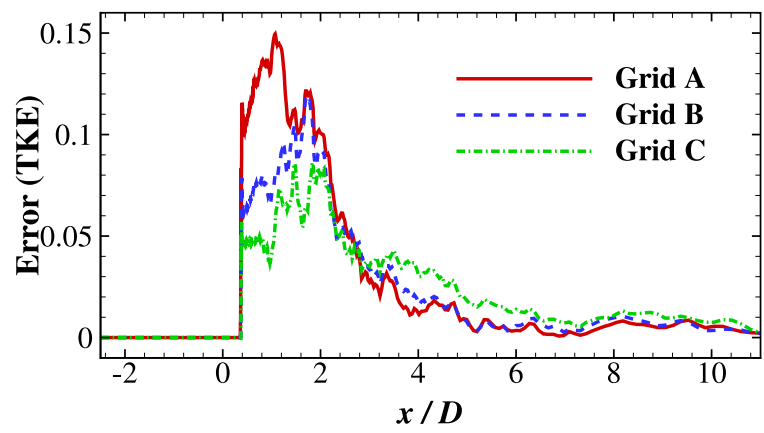

(b) Error(TKE)

Fig. 22. Errors of temporally averaged streamwise velocity and TKE along the centerline of the propeller. 


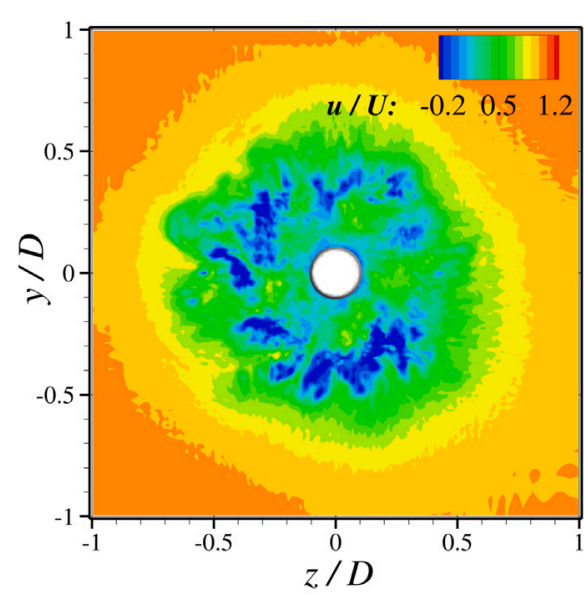

(a) Grid A

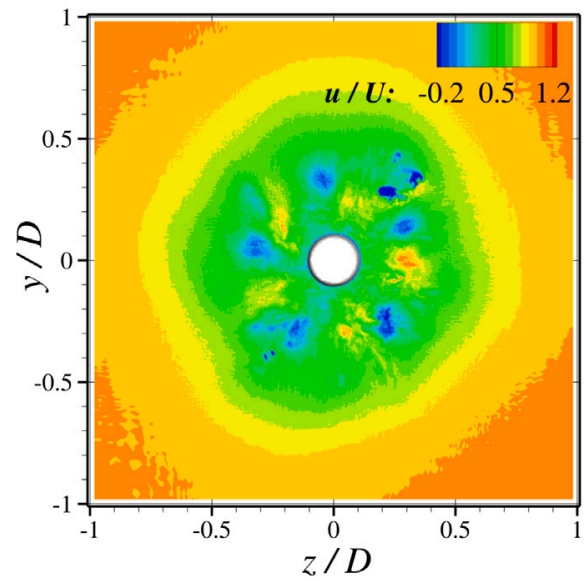

(c) Grid C

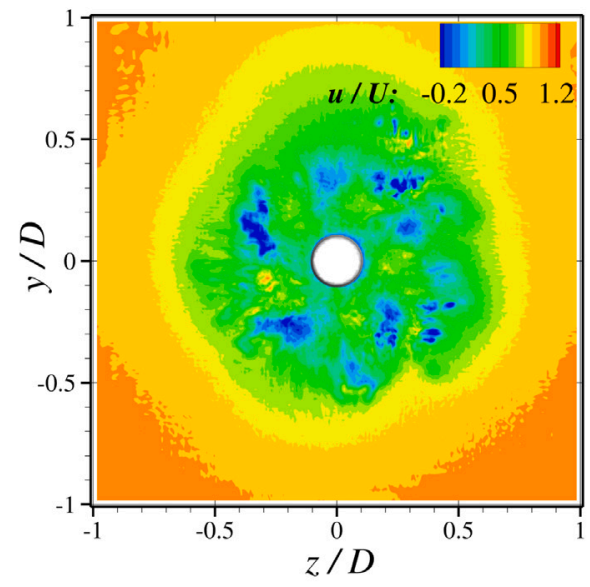

(b) Grid B

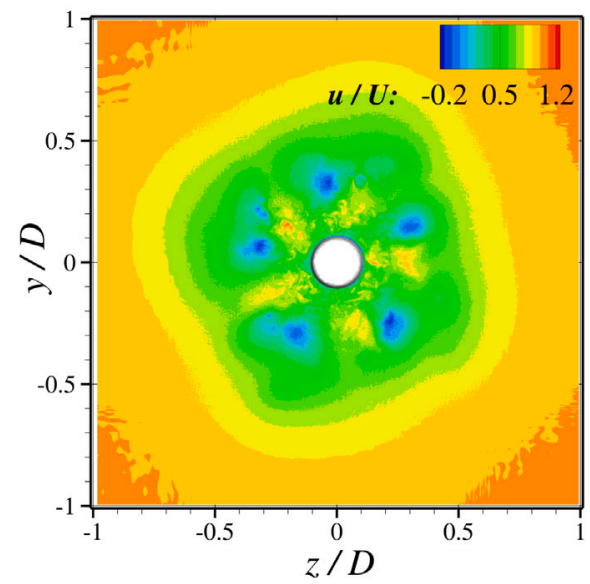

(d) Grid D

Fig. 23. Instantaneous streamwise velocity $u / U$ at $y-z$ slice located $0.2 D$ upstream of the propeller.

flow separations. To the best of our knowledge, no work has been done in the literature for the flow in the far wake of a propeller in crashback mode. This work showed that the flow in far wake is also of considerable significance, with the velocity deficit normalized by the incoming velocity $U$ in the range of 0.2 at $8 \mathrm{D}$ propeller downstream to 0.3 at $4 \mathrm{D}$ propeller downstream (Fig. 10) and TKE $/ U^{2}$ ranging from 0.01 to 0.03 at $8 \mathrm{D}$ and $4 \mathrm{D}$ propeller downstream (Fig. 12 which is similar to that of a forward propeller Liao et al., 2020), respectively. Although the coarsest grid, i.e. Grid A, is incapable to predict the flow over the blade and in the near wake, it can predict the far wake flow characteristics with reasonable accuracy.

Other than grid spacing, the employed numerical method may also cause uncertainties to the simulation results. For instance, it is not clear about the performance of the dynamic SGS model for heterogeneous turbulent flows on very coarse grids. The other uncertainty comes from the wall model, which is based on equilibrium hypothesis, such that its accuracy in predicting flow separation needs to be quantified. In addition, the accuracy of the immersed boundary method is affected by moving boundaries, which also needs to be carefully analyzed. Uncertainty analysis on how these factors affect the simulation results is very important. However, such uncertainty analysis requires a series of simulations and is beyond the scope of this work. Furthermore, these three factors interfere with each other in propeller flow simulations. It would be beneficial to carry out uncertainty analysis for each factor individually using canonical flow cases.

\section{CRediT authorship contribution statement}

Fei Liao: Writing - original draft, Visualization, Formal analysis. Xiaolei Yang: Conceptualization, Methodology, Validation, Writing - review \& editing. Shizhao Wang: Methodology, Formal analysis. Guowei He: Supervision, Project administration.

\section{Declaration of competing interest}

The authors declare that they have no known competing financial interests or personal relationships that could have appeared to influence the work reported in this paper.

\section{Acknowledgments}

This work is supported by the NSFC Basic Science Center Program for "Multiscale Problems in Nonlinear Mechanics" (No. 11988102), the National Natural Science Foundation of China (Nos. 11572331 and 91752118), and the National Numerical Wind Tunnel Project of China (NNW2018-ZT1A01). The authors would like to acknowledge the support from the Strategic Priority Research Program of the Chinese Academy of Sciences (XDB22040104), the Key Research Program of Frontier Sciences of the Chinese Academy of Sciences (QYZDJ-SSWSYS002), and the National Key Project of China (GJXM92579). 


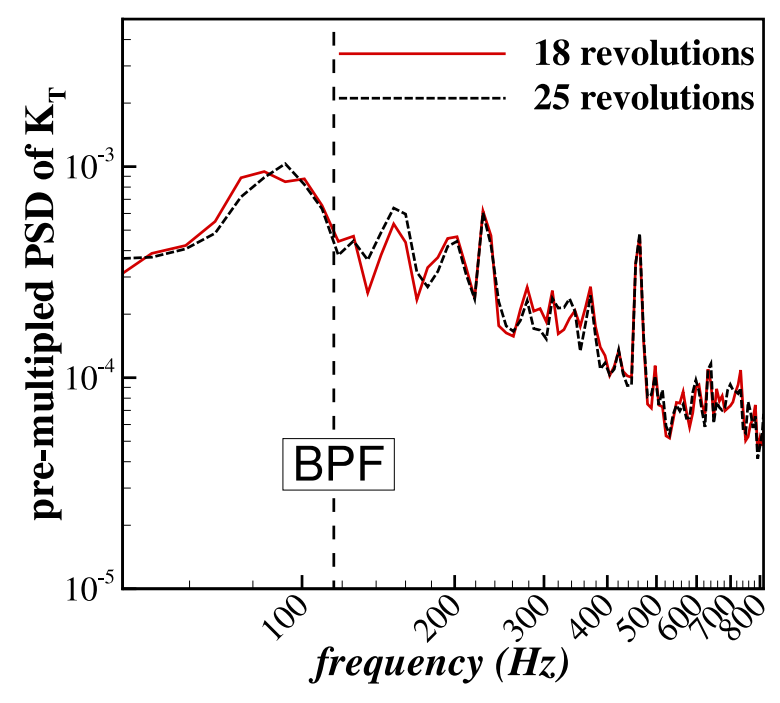

Fig. 24. Pre-multiplied PSD of $K_{T}$ computed with Grid D using different length of data.

\section{Appendix. Further discussion on thrust coefficient}

In this appendix, we explain the reason why the peak of BPF gradually decreases with the increase of grid resolution as observed in Fig. 4(a). First, we try to understand the cause for the appearance of the peak at BPF. The thrust force at a radial location is a function of the local relative incoming velocity (including velocity magnitude and direction) and the blade property (including foil type, chord length and twist angle). Under ideal conditions that the blades of the propeller are exactly the same, the velocity at the inlet is uniform, and the axis of the propeller is perfectly aligned with the inflow, which is exactly the case for the present simulation, the only possible cause for the peak at BPF is the asymmetrical oncoming flow induced by the propeller. To examine how this asymmetrical oncoming flow vary with grid resolution, we plot the instantaneous streamwise velocity contours on a $y-z$ slice located $0.2 D$ upstream of the propeller in Fig. 23. It can be observed that the oncoming flow becomes more axisymmetric as we refine the grid. Thereby, it explains the reduction of the peak of PSD at BPF with the increase of grid resolution. Moreover, the effect of different numbers of samples on computing the pre-multiplied PSD of $K_{T}$ is also tested without finding any significant differences as shown in Fig. 24.

\section{References}

Balaras, Elias, Schroeder, Seth, Posa, Antonio, 2015. Large-eddy simulations of submarine propellers. J. Ship Res. 59 (4), 227-237.

Borazjani, Iman, Ge, Liang, Sotiropoulos, Fotis, 2008. Curvilinear immersed boundary method for simulating fluid structure interaction with complex 3D rigid bodies. J. Comput. Phys. 227 (16), 7587-7620.

Brehm, Christoph, Barad, Michael F., Kiris, Cetin C., 2016. Open rotor computational aeroacoustic analysis with an immersed boundary method. In: 54th AIAA Aerospace Sciences Meeting. American Institute of Aeronautics and Astronautics, San Diego, California, USA, http://dx.doi.org/10.2514/6.2016-0815.

Chang, PA, Ebert, Michael, Young, Yin L, Liu, Zhanke, Mahesh, Krishnan, Jang, Hyunchul, Shearer, M, 2008a. Propeller forces and structural response due to crashback. In: 27th Symposium on Naval Hydrodynamics.

Chang, Peter a, Ebert, Michael, Young, Yin L., Liu, Zhanke, Mahesh, Krishnan, Jang, Hyunchul, Shearer, Matthew, 2008b. Propeller forces and structural response due to crashback. In: Proceedings of the 27 Symposium on Naval Hydrodynamics.

Ge, Liang, Sotiropoulos, Fotis, 2007. A numerical method for solving the 3D unsteady incompressible Navier-Stokes equations in curvilinear domains with complex immersed boundaries. J. Comput. Phys. 225 (2), 1782-1809.

Germano, Massimo, Piomelli, Ugo, Moin, Parviz, Cabot, William H, 1991. A dynamic subgrid-scale eddy viscosity model. Phys. Fluids A 3 (7), 1760-1765.
Griffith, Boyce E., Patankar, Neelesh A., 2020. Immersed methods for fluid-structure interaction. Annu. Rev. Fluid Mech. 52 (1), annurev-fluid-010719-060228. http: //dx.doi.org/10.1146/annurev-fluid-010719-060228.

Huang, Wei-Xi, Tian, Fang-Bao, 2019. Recent trends and progress in the immersed boundary method. Proc. Inst. Mech. Eng. C 233 (23-24), 7617-7636.

Jang, Hyunchul, Mahesh, Krishnan, 2013. Large eddy simulation of flow around a reverse rotating propeller. J. Fluid Mech. 729, 151-179.

Jessup, Stuart, Chesnakas, Chris, Fry, David, Donnelly, Martin, Black, Scott, Park, Joel, 2004. Propeller performance at extreme off design conditions. In: Proceedings of the 25th Symposium on Naval Hydrodynamics, St. John'S, Canada.

Kang, Seokkoo, 2015. An improved near-wall modeling for large-eddy simulation using immersed boundary methods. Internat. J. Numer. Methods Fluids 78 (2), 76-88. http://dx.doi.org/10.1002/fld.4008.

Kang, Seokkoo, Borazjani, Iman, Colby, Jonathan, Sotiropoulos, Fotis, 2012. Numerical simulation of 3D flow past a real-life marine hydrokinetic turbine. Adv. Water Resour. 39, 33-43.

Kang, Seokkoo, Lightbody, Anne, Hill, Craig, Sotiropoulos, Fotis, 2011. High-resolution numerical simulation of turbulence in natural waterways. Adv. Water Resour. 34 (1), 98-113.

Kang, Seokkoo, Yang, Xiaolei, Sotiropoulos, Fotis, 2014. On the onset of wake meandering for an axial flow turbine in a turbulent open channel flow. J. Fluid Mech. 744, 376-403.

Keller, Jacob, Kumar, Praveen, Mahesh, Krishnan, 2018. Examination of propeller sound production using large eddy simulation. Phys. Rev. Fluids 3 (6), 064601.

Khosronejad, Ali, Kang, Seokkoo, Borazjani, Iman, Sotiropoulos, Fotis, 2011. Curvilinear immersed boundary method for simulating coupled flow and bed morphodynamic interactions due to sediment transport phenomena. Adv. Water Resour..

Khosronejad, Ali, Le, Trung, Dewall, Petra, Bartelt, Nicole, Woldeamlak, Solomon, Yang, Xiaolei, Sotiropoulos, Fotis, 2016. High-fidelity numerical modeling of the Upper Mississippi River under extreme flood condition. Adv. Water Resour. 98 (DEC.), 97-113.

Kumar, Praveen, Mahesh, Krishnan, 2016. Towards large eddy simulation of hullattached propeller in crashback. In: Proceedings of the 31st Symposium on Naval Hydrodynamics. Monterey, USA.

Kumar, Praveen, Mahesh, Krishnan, 2017. Large eddy simulation of propeller wake instabilities. J. Fluid Mech. 814, 361-396.

Liao, Fei, Wang, Shizhao, Yang, Xiaolei, He, Guowei, 2020. A simulation-based actuator surface parameterization for large-eddy simulation of propeller wakes. Ocean Eng. 199, 107023. http://dx.doi.org/10.1016/j.oceaneng.2020.107023.

Ma, Ming, Huang, Wei-Xi, Xu, Chun-Xiao, 2019. A dynamic wall model for large eddy simulation of turbulent flow over complex/moving boundaries based on the immersed boundary method. Phys. Fluids 31 (11).

Nicoud, Franck, Ducros, Frédéric, 1999. Subgrid-scale stress modelling based on the square of the velocity gradient tensor. Flow Turbul. Combust. 62 (3), 183-200.

Posa, Antonio, Balaras, Elias, 2018. Large-Eddy Simulations of a notional submarine in towed and self-propelled configurations. Comput. \& Fluids 165, 116-126.

Posa, Antonio, Broglia, Riccardo, Balaras, Elias, 2019b. LES study of the wake features of a propeller in presence of an upstream rudder. Comput. \& Fluids 192, 104247.

Posa, Antonio, Broglia, Riccardo, Felli, Mario, Falchi, Massimo, Balaras, Elias, 2019a. Characterization of the wake of a submarine propeller via large-eddy simulation. Comput. \& Fluids 184, 138-152.

Roman, F., Armenio, V., Fröhlich, J., 2009. A simple wall-layer model for large eddy simulation with immersed boundary method. Phys. Fluids 21 (10), 101701. http://dx.doi.org/10.1063/1.3245294.

Rosenfeld, Moshe, 1994. Grid refinement test of time-periodic flows over bluff bodies. Comput. \& Fluids 23 (5), 693-709.

Roy, Somnath, De, Ashoke, Balaras, Elias (Eds.), 2020. Immersed Boundary Method: Development and Applications. In: Computational Methods in Engineering \& the Sciences, Springer Singapore, Singapore, http://dx.doi.org/10.1007/978-981-153940-4.

Shi, Beiji, Yang, Xiaolei, Jin, Guodong, He, Guowei, Wang, Shizhao, 2019. Wallmodeling for large-eddy simulation of flows around an axisymmetric body using the diffuse-interface immersed boundary method. Appl. Math. Mech. 40 (3), 305-320. http://dx.doi.org/10.1007/s10483-019-2425-6.

Smargorinsky, Joseph, 1963. General circulation experiments with the primitive equations. Mon. Weather Rev. 91 (3), 99-164.

Sotiropoulos, Fotis, Yang, Xiaolei, 2014. Immersed boundary methods for simulating fluid-structure interaction. Prog. Aerosp. Sci. 65, 1-21.

Tessicini, F, Iaccarino, G, Fatica, M, Wang, M, Verzicco, R, 2002. Wall Modeling for Large-Eddy Simulation Using an Immersed Boundary Method. Annual Research Briefs, Center for Turbulence Research, Stanford, pp. 181-187.

Toosi, Siavash, 2019. Error Estimation, Grid Selection and Convergence Verification in Large Eddy Simulation (Ph.D. thesis). University of Maryland, College Park.

Verma, A., Jang, H., Mahesh, Krishnan, 2012. The effect of an upstream hull on a propeller in reverse rotation. J. Fluid Mech. 704, 61-88.

Vysohlid, Martin, Mahesh, Krishnan, 2006. Large eddy simulation of crashback in marine propellers, in: 44th AIAA Aerospace Sciences Meeting and Exhibit, p. 1415.

Vysohlid, Martin, Mahesh, Krishnan, 2007. Understanding crashback in marine propellers using an unsteady actuator disk model. In: 45th AIAA Aerospace Sciences Meeting and Exhibit. p. 918. 
Wang, Shizhao, Shi, Beiji, Li, Yuhang, He, Guowei, 2016. A large eddy simulation of flows around an underwater vehicle model using an immersed boundary method. Theor. Appl. Mech. Lett. 6 (6), 302-305.

Wang, Shizhao, Zhang, Xing, 2011. An immersed boundary method based on discrete stream function formulation for two- and three-dimensional incompressible flows. J. Comput. Phys. 230 (9), 3479-3499.

Yang, Xiaolei, Khosronejad, Ali, Sotiropoulos, Fotis, 2017. Large-eddy simulation of a hydrokinetic turbine mounted on an erodible bed. Renew. Energy 113 (dec.), 1419-1433.
Yang, Xiaolei, Sotiropoulos, Fotis, 2018. A new class of actuator surface models for wind turbines. Wind Energy 21 (5), 285-302.

Yang, Xiaolei, Sotiropoulos, Fotis, 2019. A review on the meandering of wind turbine wakes. Energies 12 (24), 4725.

Yang, Xiaolei, Sotiropoulos, Fotis, Conzemius, Robert J, Wachtler, John N, Strong, Mike B, 2015. Large-eddy simulation of turbulent flow past wind turbines/farms: the Virtual Wind Simulator (VWiS). Wind Energy 18 (12), 2025-2045. 